\title{
Sur la transformation d'Abel-Radon des courants localement résiduels
}

\author{
BRUNO FABRE
}

\begin{abstract}
After recalling the definitions of the Abel-Radon transformation of currents and of locally residual currents, we show that the Abel-Radon transform $\mathcal{R}(\alpha)$ of a locally residual current $\alpha$ remains locally residual. Then a theorem of P. Griffiths, G. Henkin and M. Passare (cf. [7], [9] and [10]) can be formulated as follows : Let $U$ be a domain of the Grassmannian variety $G(p, N)$ of complex $p$-planes in $\mathbb{P}^{N}, U^{*}:=\cup_{t \in U} H_{t}$ be the corresponding linearly p-concave domain of $\mathbb{P}^{N}$, and $\alpha$ be a locally residual current of bidegree $(N, p)$. Suppose that the meromorphic $n$-form $\mathcal{R}(\alpha)$ extends meromorphically to a greater domain $\tilde{U}$ of $G(p, N)$. If $\alpha$ is of type $\omega \wedge[T]$, with $T$ an analytic subvariety of pure codimension $p$ in $U^{*}$, and $\omega$ a meromorphic (resp. regular) $q$-form $(q>0)$ on $T$, then $\alpha$ extends in a unique way as a locally residual current to the domain $\tilde{U}^{*}:=\cup_{t \in \tilde{U}} H_{t}$. In particular, if $\mathcal{R}(\alpha)=0$, then $\alpha$ extends as a $\bar{\partial}$-closed residual current on $\mathbb{P}^{N}$. We show in this note that this theorem remains valid for an arbitrary residual current of bidegree $(N, p)$, in the particular case where $p=1$.
\end{abstract}

Mathematics Subject Classification (2000): 32C30 (primary); 44A12 (secondary).

\section{Introduction}

On introduit dans la section suivante une classe particulière de courants sur un espace analytique $X$ de dimension pure $n$, appelés localement résiduels, qui s'écrivent localement comme des courants résiduels suivant la définition de [2], généralisant ainsi les courants de la forme $\omega \wedge[Y]$, avec $\omega$ une forme méromorphe sur un sous-ensemble analytique $Y \subset X$ de dimension pure. La définition est proche de celle donnée par [3], mais on accepte ici les courants non $\bar{\partial}$-fermés. On rappelle quelques propriétés fondamentales de ces courants, dont la caractérisation de J.-E. Björk ([1]). On montre dans le cas particulier du degré maximal $(n, n)$ que le courant se calcule par le résidu ponctuel de Grothendieck; dans le cas où $X=\mathbb{C}^{n}$, et que le support est fini, on montre que le courant s'annule ssi il s'annule sur tous les polynômes. 
Dans une troisième section, on étudie la transformation d'Abel-Radon des courants dans un cadre général. Dans les deux premiers paragraphes, on étudie les images directe et inverse d'un courant par une application holomorphe, qu'on peut voir comme des cas particuliers de la transformation d'Abel-Radon. Puis on rappelle la définition de la transformation d'Abel-Radon donnée dans [6] : étant donné une famille de $p$-cycles complexes $\left(H_{t}\right)_{t \in T}$ sur un espace analytique $X$, associée à un espace de paramètres $T$ et à une sous-espace d'incidence $I \subset T \times X$, la transformation d'Abel-Radon $\mathcal{R}$ d'un courant $\alpha$ est définie par $\mathcal{R}(\alpha):=\left(p_{1}\right)_{*}\left(p_{2}^{*}(\alpha)\right)$, où $p_{1}: I \rightarrow T$ et $p_{2}: I \rightarrow X$ sont les projections naturelles, supposées respectivement propres et submersives. Si $\alpha$ est un courant de bidegré $(r, s)$ sur $X$, alors $\mathcal{R}(\alpha)$ est un courant de bidegré $(r-p, s-p)$, (avec $p=\operatorname{dim}(I)-\operatorname{dim}(T))$. De plus, $\mathcal{R}$ commute avec les opérateurs $d, \partial, \bar{\partial}$. Si $\alpha$ est une forme lisse de degré $2 p$, on a : $\mathcal{R}(\alpha)=\int_{H_{t}} \alpha$. On montre pour les courants localement résiduels une propriété de stabilité pour la transformation d'Abel-Radon :

Théorème 1.1. Si $\alpha$ est un courant localement résiduel sur $X$, alors $\mathcal{R}(\alpha)$ est encore un courant localement résiduel sur $T$. En particulier, $\mathcal{R}$ transforme un courant localement résiduel de bidegré $(q+p, p)$ en une q-forme méromorphe $\operatorname{sur} T$.

Dans une quatrième section, on rappelle quelques résultats de prolongement analytique qu'on utilisera dans les sections suivantes.

La cinquième section est consacrée à l'étude du cas particulier suivant de la transformation d'Abel-Radon : $X=T \times \mathbb{P}^{1}$, et la famille de cycles est l'ensemble des fibres $\left(F_{t}=\phi^{-1}(t)\right)_{t \in T}$, avec $\phi: X \rightarrow T$ la projection standard. On définit alors les traces d'un courant $\alpha$ localement résiduel de bidegré $(q+1,1)$, dont le support $S$ n'a pas de composante verticale (i.e. $S$, qui est une hypersurface analytique de $X$, ne contient pas d'hypersurface de la forme $\left.\phi^{-1}(H), H \subset T\right)$ et rencontre $T_{\infty}:=T \times\{\infty\}$ proprement, en posant, pour $k \in \mathbb{N}: v_{k}:=\phi_{*}\left(\alpha y^{k}\right), w_{k}:=\phi_{*}\left(\alpha y^{k} \wedge d y\right)$, qui définissent deux suites de formes méromorphes sur $T$, de degrés respectifs $q-1$ et $q$. On montre alors que $\alpha$ peut se reconstruire à partir de ses traces. Plus précisément :

Théorème 1.2. (des traces). Soit $U$ un ouvert de $T$, et $\alpha$ un courant localement résiduel de bidegré $(q, 1)$ sur $U \times \mathbb{P}^{1}$, dont le support $S$ est sans composante verticale et coupe $T_{\infty}$ proprement. Alors, $(\forall k \in \mathbb{N}), v_{k}=w_{k}=0$ implique $\alpha=0$. Si pour tout $k \in \mathbb{N} v_{k}$ et $w_{k}$ sont holomorphes, $\alpha$ est $\bar{\partial}$-fermé. De plus, supposons que pour tout $k \in \mathbb{N}$, $v_{k}$ et $w_{k}$ se prolongent méromorphiquement dans T. Alors, $\alpha$ se prolonge de manière unique en un courant localement résiduel a de bidegré $(q, 1)$ sur $X$ dont le support $\tilde{S}$ est sans composante verticale. De plus, si $\alpha \bar{\partial}$-fermé sur $\phi^{-1}(U)$, et que ses traces $v_{k}, w_{k} k \in \mathbb{N}$ se prolongent holomorphiquement sur $X$, alors $\tilde{\alpha}$ reste $\bar{\partial}$-fermé sur $Y$.

On étudie dans la sixième section le cas particulier de la transformation d'AbelRadon où l'espace des paramètres est un domaine $U$ de la grassmannienne $G(p, N)$ des $p$-plans complexes, et où l'espace $X$ est l'ouvert $U^{*}=\cup_{t \in U} H_{t}$ correspondant de l'espace projectif complexe $\mathbb{P}^{N}$, appelé linéairement p-concave 
car étant réunion de $p$-plans complexes. On appelle ce cas particulier la transformation de Radon-Martineau, la transformation du même nom donnée dans [11] correspondant au cas particulier $p=N-1$. Le problème étudié dans cette section peut alors se formuler de la manière suivante. Soit $\alpha$ un courant localement résiduel de bidegré $(q+p, p)(0<q \leq N-p)$ sur l'ouvert linéairement $p$-concave $U^{*}=\cup_{t \in U} H_{t}$, de sorte que $\mathcal{R}(\alpha)$ est une $q$-forme méromorphe sur $U$. Supposons que $\mathcal{R}(\alpha)$ se prolonge méromorphiquement sur un domaine $\tilde{U}$ contenant $U$. G. Henkin et M. Passare ont montré dans [10] que, sous la condition que le courant $\alpha$ est de la forme $\omega \wedge[Y]$, où $\omega$ est une $q$-forme méromorphe sur un sous-ensemble analytique $Y$ de $U$ de codimension $p$, et que $\tilde{U}$ est projectivement convexe, alors $\alpha$ se prolonge à $\tilde{U}^{*}:=\cup_{t \in \tilde{U}^{*}} H_{t}$ comme courant localement résiduel. L'énoncé resterait-il valide pour un courant localement résiduel de bidegré $(q+p, p)$ arbitraire, et en supposant juste l'ouvert $\tilde{U}$ connexe? Le problème d'une généralisation du théorème d'Henkin-Passare au cas d'un courant localement résiduel "à support non-réduit" avait déjà été formulé dans [11] pour $N=2$. On donne dans cette note une réponse positive dans le cas de la codimension $p=1$. On suppose dans ce qui suit $p=1$.

Théorème 1.3. (de Griffiths généralisé). On se donne $\alpha$ un courant localement résiduel de bidegré $(q+1,1), 0<q \leq N-1$ sur $U^{*}:=\cup_{t \in U} H_{t}$. Si $\mathcal{R}(\alpha)=0, \alpha$ se prolonge de manière unique à un courant localement résiduel $\tilde{\alpha}$ sur $\mathbb{P}^{N}$; on a de plus $\alpha=\bar{\partial}[\tilde{\Psi}]$ pour une $(q+1)$-forme rationnelle $\Psi$ sur $\mathbb{P}^{N}$.

Théorème 1.4. (d'Henkin-Passare généralisé). On se donne un courant localement résiduel (resp. localement résiduel et $\bar{\partial}$-fermé) $\alpha$ de bidegré $(N, 1)$ dans $U^{*}$. On suppose que $\mathcal{R}(\alpha)$, qui est méromorphe (resp. holomorphe) sur $U$, se prolonge méromorphiquement (resp. holomorphiquement) dans un domaine $\tilde{U}$ contenant $U$. Alors $\alpha$ se prolonge de manière unique dans $\tilde{U}^{*}$ comme courant localement résiduel (resp. localement résiduel $\bar{\partial}$-fermé).

REMERCIEMENTS. Je remercie le professeur G. Tomassini pour son acceuil à l'Ecole Normale Supérieure de Pise et pour les discussions encourageantes qu'il a bien voulu m'accorder ; ainsi que le professeur M. Giaquinta, pour son invitation au Centro de Giorgi, où j'ai pu terminer la rédaction de cet article.

\section{Sur les courants localements résiduels}

\subsection{Les courants sur un espace analytique réduit}

Soit $X$ un espace analytique réduit. On a donc pour tout point $x \in X$ un isomorphisme analytique (appelé carte) $\phi: U \rightarrow V$ d'un voisinage ouvert $U$ de $x$ sur un sous-ensemble analytique $V$ d'un ouvert $W$ de $\mathbb{C}^{n} ; x$ est régulier ssi on peut prendre $V=W$. 
On a de manière naturelle des faisceaux sur $X$ de germes de $p$-formes différentielles (à valeurs complexes) de classe $\mathcal{C}^{k}$ (resp. lisses, holomorphes) sur l'ouvert $\operatorname{Reg}(X)$ de $X$ formé des points réguliers. On définit des sous-faisceaux de formes différentielles de classe $\mathcal{C}^{k}$ (resp. lisses, holomorphes) sur $X$ en disant qu'un germe de forme en $x \Psi_{x}$ est de classe $\mathcal{C}^{k}$ (resp. lisse, holomorphe) si pour une carte de $X$ en $x \phi: U \rightarrow V \subset W$, le germe de forme en $\phi(x) \in V \phi_{*}\left(\Psi_{x}\right)$ provient d'un germe de $p$-forme de classe $\mathcal{C}^{k}$ (resp. lisse, holomorphe) en $\phi(x)$ dans $W$. Sur $W$, on a une topologie naturelle sur les formes lisses, resp. lisses à support compact (cf. [17]). Un courant sur $V \subset W$ (resp. un courant à support compact) est alors une forme linéaire continue sur les formes lisses $\grave{a}$ support compact (resp. sur les formes lisses) de $W$, qui s'annule sur les formes qui s'annulent sur $V$ (i.e. dont la restriction à $\operatorname{Reg}(V)$ s'annule). Etant donnée une carte $\phi: U \rightarrow V \subset W$, un courant sur $U$ est une forme linéaire $\alpha$ sur les formes lisses à support compact de $U$, telle que $\beta(\psi):=\alpha\left(\phi^{*}(\psi)\right)$ définit un courant $V$. Un courant sur $X$ est alors une forme linéaire sur les formes lisses à support compact, qui pour chaque carte $\phi: U \rightarrow V \subset W$ définit par restriction un courant sur $U$. Un courant $\alpha$ à support compact $S$ s'étend de manière unique en une forme linéaire sur les formes lisses (à support quelconque), en posant : $\alpha(\Psi):=\alpha(\chi \Psi)$, avec $\chi$ une fonction plateau lisse à support compact, égale à 1 dans un voisinage de $S$. Toute $r$-forme lisse sur $X$ se décompose de manière unique en $r$-formes de bidegré $(p, q)$, avec $p+q=r: \omega:=\sum_{p+q} \omega_{p, q}$. Un courant sur $X$ est de dimension $r$, ou de bidimension $(p, q)$ s'il s'annule sur les formes de degré $\neq r$ (resp. de bidegré $\neq(p, q)$ ). Si $X$ est de dimension pure (complexe) $n$, ce qu'on supposera par la suite, on dira que ce courant est de degré $2 n-r$ (resp. de bidegré $(n-p, n-q)$ ). Une distribution est un courant de degré 0 . On peut multiplier un courant $T$ par une forme lisse $\psi$, en posant $T \wedge \psi(\Psi):=T(\psi \wedge \Psi)$. On obtient ainsi une structure de module sur les formes lisses. Sur $\operatorname{Reg}(X)$, ce module est libre, de sorte qu'on peut voir un courant de degré $r$ comme une $r$-forme à coefficients distributions. On en déduit que tout courant $T$ de degré $r$ se décompose de manière unique sur $\operatorname{Reg}(X)$ sous la forme $T=\sum_{p+q=r} T_{p, q}$, avec $T_{p, q}$ un courant de bidegré $(p, q)$, i.e. une $(p, q)$-forme à coefficients distributions. L'opérateur de différentiation $d$ se décompose alors en somme : $d=\partial+\bar{\partial}$, de sorte que $\partial$ associe à toute forme (resp. courant) de bidegré $(p, q)$ une forme (resp. courant) de bidegré $(p+1, q)$, et $\bar{\partial}$ associe à toute forme (resp. courant) de bidegré $(p, q)$ une forme (resp. courant) de bidegré $(p, q+1)$.

\subsection{Valeur principale et résidu}

Soit $X$ un espace analytique réduit de dimension pure. Etant donné une fonction méromorphe $f$ sur $X, f$ est holomorphe en dehors d'une hypersurface $T$ de $X$, qu'on appelle son lieu polaire. On a une distribution $[f]$ bien définie en dehors de $T$. Si $f$ est localement intégrable, cette distribution s'étend de manière canonique en distribution sur $X$, d'ordre nul. Si $f$ n'est pas localement intégrable, le fait que $f$ est méromorphe permet de montrer l'existence d'un prolongement à travers $T$ de $f$, comme distribution. Mais on ne sait pas a priori 
faire un choix "canonique" parmi tous les prolongements possibles. Hererra et Lieberman ont montré dans [12] le théorème suivant :

Lemme 2.1. Soit $g$ une fonction holomorphe sur un ouvert $U \subset X$, telle que $\operatorname{Sing}(X) \cup T \subset\{g=0\}$. Alors les limites $\lim _{\epsilon \rightarrow 0} \int_{|g|>\epsilon} f \phi$ et $\lim _{\epsilon \rightarrow 0} \int_{|g|=\epsilon} f \phi$ existent pour $\phi$ à support compact dans $U$, et définissent des courants sur $U$, qui ne dépendent pas du choix de g. On les note respectivement $[f]_{U}$ et $\operatorname{Res}_{T \cap U}(f)$. De plus, $\operatorname{Res}_{T \cap U}(f)=\bar{\partial}[f]_{U}$.

Le fait que la définition de $[f]$ ne dépend pas du choix de $g$ montre que sur $U \cap V,[f]_{U}=[f]_{V}$; on peut ainsi définir par un recouvrement ouvert un courant sur $X$, noté $[f]$, qui ne dépend pas du recouvrement choisi. On appelle la distribution $[f]$ la valeur principale de $f$.

On peut déduire de ce qui précède que $[f+g]=[f]+[g]$ et si $h$ est une fonction holomorphe, $[h f]=h[f]$. De plus, on peut montrer que si $D$ est une dérivation holomorphe, et $f$ une fonction méromorphe, alors $D f$ est encore méromorphe, et $[D f]=D[f]$.

Soit $\chi(x): \mathbb{R}^{+} \rightarrow \mathbb{R}^{+}$une fonction lisse et croissante telle que $\chi(x)=0$ au voisinage de 0 et $\chi(x)=1$ pour $x \geq 1$, et soit $g$ une fonction holomorphe sur $U$ telle que $(T \cup \operatorname{Sing}(X)) \cap U \subset\{g=0\}$. Alors, on peut déduire du lemme précédent : $\lim _{\epsilon \rightarrow 0} \chi(|g| / \epsilon)[f]=[f]$ dans l'espace des distributions de $U$.

Définition 2.2. Un courant $\alpha$ sur $X$, de support $S$, est dit d'extension standard si : pour toute fonction holomorphe $g$ sur un domaine $U$ de $X$, qui ne s'annule sur aucun ouvert de $S$, et pour toute fonction lisse $\chi(x): \mathbb{R}^{+} \rightarrow \mathbb{R}^{+}$croissante telle que $\chi(x)=0$ au voisinage de 0 et $\chi(x)=1$ pour $x \geq 1, \lim _{\epsilon \rightarrow 0} \chi(|g| / \epsilon) \alpha=\alpha$ dans $U$.

Alors, ce qui précède montre que la distribution valeur principale $[f]$ associée à une fonction méromorphe $f$ est d'extension standard.

Soit maintenant $\Psi$ une $q$-forme méromorphe sur $X$. Pour $x \in X$, on écrit pour le germe en $x$ de $q$-forme méromorphe $\Psi_{x} \Psi_{x}:=\omega_{x} / g_{x}$, avec $\omega_{x}$ une $q$ forme méromorphe et $g_{x}$ une fonction holomorphe ; Alors, on associe le germe de courant en $x:\left[\Psi_{x}\right]:=\omega_{x}\left[1 / g_{x}\right]$; on vérifie que le courant ne dépend pas du réprésentant choisi ; d'où un courant valeur principale $[\Psi]$ sur $X$.

On peut déduire de ce qui précède :

Lemme 2.3. Soit $\alpha$ un courant de bidegré $(q, 0)$ sur $X$. Supposons que:

1. $\alpha$ est $\bar{\partial}$-fermé en dehors d'une hypersurface complexe $T$.

2. $\alpha$ est d'extension standard sur $X$.

Alors, $\alpha=[\omega]$ pour une certaine q-forme méromorphe $\omega$ sur $X$.

Soit $\Psi$ une $q$-forme holomorphe en dehors d'une hypersurface $T$ de $X$. Si $\Psi$ se prolonge en une forme méromorphe $\tilde{\Psi}$ sur $X$, le courant $[\tilde{\Psi}]$ définit un prolongement du courant défini par $\Psi$ sur $X \backslash T$. G. Henkin et M. Passare ont montré dans [10] que :

Lemme 2.4. Si le courant $[\Psi]$ sur $X \backslash T$ se prolonge en un courant $\alpha$ sur $X$, alors $\Psi$ admet un prolongement méromorphe $\tilde{\Psi}$ sur $X$. De plus, si $\bar{\partial} \alpha=0$, alors $\bar{\partial}[\tilde{\Psi}]=0$. 
Définition 2.5. $\Psi$ sur $X$ est dite régulière (ou encore parfois abélienne si $X$ est algébrique) si le courant $[\Psi]$ sur $X$ est $\bar{\partial}$-fermé.

Remarque. Il existe en général des courants $\bar{\partial}$-fermés de bidegré $(q, 0)$ à support dans $\operatorname{Sing}(X)$.

\subsection{Courants résiduels de codimension quelconque}

Supposons maintenant qu'on ait $p+1$ hypersurfaces analytiques $Y_{1}, \ldots, Y_{p+1}$ dans $X$ qui se coupent proprement, i.e. telles que $Y_{1} \cap \cdots \cap Y_{p+1}$ soit de codimension pure $p+1$. On se donne une $q$-forme méromorphe $\Psi$ dont le lieu polaire est contenu dans $Y_{1} \cup \cdots \cup Y_{p+1}$. M. Herrera et N. Coleff on montré dans [2] le résultat suivant :

Lemme 2.6. Soit $U$ un ouvert de $X$, et $\left(f_{1}, \ldots, f_{p+1}\right)$ une suite régulière de fonctions holomorphes sur $U$ (de sorte que $f_{1}=\cdots=f_{p+1}=0$ est un sous-ensemble analytique de $U$ de codimension $p+1)$, telles que $Y_{i} \cap U \subset\left\{f_{i}=0\right\}(1 \leq i \leq p+1)$. Pour une forme $\phi$ lisse à support compact dans $U$, la limite suivante existe :

$$
\lim _{t \rightarrow 0} \int_{\left|f_{p+1}\right| \geq \epsilon_{p+1}(t),\left|f_{1}\right|=\epsilon_{1}(t), \ldots,\left|f_{p}\right|=\epsilon_{p}(t)} \Psi \wedge \phi
$$

pour n'importe quel "chemin" $[0,1] \rightarrow \mathbb{R}_{+}^{p+1}, t \mapsto\left(\epsilon_{1}(t), \ldots, \epsilon_{p+1}(t)\right)$ admissible, i.e. tel que $\lim _{t \rightarrow 0} \epsilon_{i}(t)=\lim _{t \rightarrow 0} \epsilon_{i+1}(t) / \epsilon_{i}(t)^{k}=0$ pour $k \in \mathbb{N}$ et $1 \leq i \leq p$. Si le lieu polaire de $\Psi$ est contenu dans $Y_{1} \cup \cdots \cup Y_{p}$, cette limite peut aussi s'écrire :

$$
\lim _{t \rightarrow 0} \int_{\left|f_{1}\right|=\epsilon_{1}(t), \ldots,\left|f_{p}\right|=\epsilon_{p}(t)} \Psi \wedge \phi .
$$

De plus, ces limites ne dépendent pas du chemin admissible, ni du choix des fonctions $f_{i}$, et définissent un courant sur $U$, de bidegré $(q, p)$. De plus, ce courant est nul si $\Psi$ est à lieu polaire contenu dans $Y_{2} \cup \cdots \cup Y_{p+1}$.

On voit qu'en un point $x \notin Y_{1} \cap \cdots \cap Y_{p} \cap U$, pour un certain $i \in\{1, \ldots, p\}$, $x \notin Y_{i}$, et alors $\Psi$ en ce point n'a pas de pôle sur $Y_{i}$; le courant défini ci-dessus a donc un support contenu dans $Y_{1} \cap \cdots \cap Y_{p} \cap U$. Etant donné un recouvrement ouvert $\left(U_{i}\right)$ de $X$, tel qu'on peut définir sur chaque $U_{i}$ une suite régulière $\left(f_{1}, \ldots, f_{p+1}\right)$ comme ci-dessus, on voit que les différents courants obtenus sur chaque ouvert se recollent pour définir un courant sur $X$, qu'on note $\operatorname{Res}_{Y_{1}, \ldots, Y_{p}} \Psi$, et qu'on appelle courant résiduel. Son support est visiblement contenu dans $Y_{1} \cap \cdots \cap Y_{p}$. Un tel courant est de bidegré $(q, p) ; p$ s'appelle la codimension du courant.

M. Passare a introduit dans [14] une autre notation utile pour ces courants. Supposons qu'on puisse trouver globalement une suite régulière $\left(f_{1}, \ldots, f_{p}\right)$ (resp. $\left.\left(f_{1}, \ldots, f_{p+1}\right)\right)$ de fonctions holomorphes sur $X$ telles que $Y_{i}=\left\{f_{i}=0\right\}$, et que $\Psi$ s'écrive sous la forme $\phi /\left(f_{1} \ldots f_{p}\right)$ (resp. $\left.\phi /\left(f_{1} \ldots f_{p} f_{p+1}\right)\right)$, avec $\phi$ une $q$-forme holomorphe sur $X$. Alors le courant $\operatorname{Res}_{Y_{1}, \ldots, Y_{p}}(\Psi)$ se note aussi : 
$\phi \bar{\partial}\left[1 / f_{1}\right] \wedge \cdots \wedge \bar{\partial}\left[1 / f_{p}\right]$ (resp. $\phi / f_{p+1} \bar{\partial}\left[1 / f_{1}\right] \wedge \cdots \wedge \bar{\partial}\left[1 / f_{p}\right]$ ). Remarquons que tout courant résiduel s'écrit localement sous la forme $\phi / f_{p+1} \bar{\partial}\left[1 / f_{1}\right] \wedge \cdots \wedge$ $\bar{\partial}\left[1 / f_{p}\right]$. En effet, sur $U$, supposons que le courant $\operatorname{Res}_{Y_{1}, \ldots, Y_{p}}(\Psi)$ s'écrive avec une suite régulière $\left(f_{1}, \ldots, f_{p}\right)$, telle que $Y_{i} \subset Y_{i}^{\prime}:=\left\{f_{i}=0\right\}$. Alors, on a : $\operatorname{Res}_{Y_{1}, \ldots, Y_{p}}(\Psi)=\operatorname{Res}_{Y_{1}^{\prime}, \ldots, Y_{p}^{\prime}}(\Psi)$, puisqu'on a vu que la limite ne dépend pas du choix des $f_{i}$. Mais $\Psi$ a un lieu polaire contenu dans $Y_{1} \cup \cdots \cup Y_{p+1}$; par conséquent, pour un certain entier $k,\left(f_{1} \ldots f_{p+1}\right)^{k} \Psi$ se prolonge sur $U$ en une $q$-forme holomorphe $\phi$. Alors, $\operatorname{Res}_{Y_{1}^{\prime}, \ldots, Y_{p}^{\prime}}(\Psi)=\operatorname{Res}_{Y_{1}^{\prime}, \ldots, Y_{p}^{\prime}}\left(\phi /\left(f_{1}^{k} \ldots f_{p+1}^{k}\right)\right.$, ou encore d'après la notation précédente : $\phi / f_{p+1}^{k} \bar{\partial}\left[1 / f_{1}^{k}\right] \wedge \cdots \wedge \bar{\partial}\left[1 / f_{p}^{k}\right]$.

On a alors :

$$
\bar{\partial}\left(1 / f_{p+1} \bar{\partial}\left[1 / f_{1}\right] \wedge \cdots \wedge \bar{\partial}\left[1 / f_{p}\right]\right)=\bar{\partial}\left[1 / f_{p+1}\right] \wedge \bar{\partial}\left[1 / f_{1}\right] \wedge \cdots \wedge \bar{\partial}\left[1 / f_{p}\right]
$$

En particulier, $\bar{\partial}\left[1 / f_{1}\right] \wedge \cdots \wedge \bar{\partial}\left[1 / f_{p}\right]$ est $\bar{\partial}$-exact. De plus, toujours d'après [14] :

Lemme 2.7. Etant donné un germe en $x \in X$ de fonction holomorphe $h$, $h\left[1 / f_{p+1}\right] \bar{\partial}\left[1 / f_{1}\right] \wedge \cdots \wedge \bar{\partial}\left[1 / f_{p}\right]=0$ ssi h appartient à l'idéal de $\mathcal{O}_{x}$ engendré en $x$ par $f_{1}, \ldots, f_{p}$.

Remarque. On sait que sous certaines conditions relatives au "front d'onde", on peut multiplier les distributions. Il serait intéressant de savoir si, dans le cas où $f_{1}, \ldots, f_{p}, g$ forment une suite régulière de fonctions holomorphes, on peut multiplier les distributions $\partial_{\overline{z_{1}}}\left[1 / f_{1}\right], \ldots, \partial_{\overline{z_{p}}}\left[1 / f_{p}\right],[1 / g]$. Les notations précédentes pour les courants résiduels se trouveraient alors parfaitement justifiées.

On a les propriétés suivantes ([3]) :

1. $\operatorname{Res}_{Y_{1}, \ldots, Y_{p}} \Psi$ est $\bar{\partial}$-fermé ssi on peut choisir $\Psi$ à pôle contenu dans $Y_{1} \cup$ $\cdots \cup Y_{p}$.

2. On a $\operatorname{Res}_{Y_{1}, \ldots, Y_{p+1}} \Psi=0$ ssi $\Psi=\sum_{i=1}^{p+1} \Psi_{i}$, avec $\Psi_{i}$ une $q$-forme méromorphe à pôle contenu dans $Y_{1} \cup \cdots \cup Y_{i-1} \cup Y_{i+1} \cup \cdots \cup Y_{p+1}$.

3. $\bar{\partial} \operatorname{Res}_{Y_{1}, \ldots, Y_{p}}(\Psi)=\operatorname{Res}_{Y_{1}, \ldots, Y_{p+1}}(\Psi)$.

En particulier, on en déduit qu'un courant résiduel $\bar{\partial}$-fermé est toujours $\bar{\partial}$-exact : $\operatorname{Res}_{Y_{1}, \ldots, Y_{p}}(\Psi)=\bar{\partial} \operatorname{Res}_{Y_{1}, \ldots, Y_{p-1}}(\Psi)$.

\subsection{Courants localement résiduels}

Définition 2.8. Un courant localement résiduel de codimension p est un courant s'écrivant dans le voisinage de tout point comme courant résiduel de codimension $p$; en particulier, il s'écrit localement sous la forme :

$$
\omega \wedge[1 / g] \bar{\partial}\left[1 / f_{1}\right] \wedge \cdots \wedge \bar{\partial}\left[1 / f_{p}\right]
$$

où $\omega$ est une q-forme holomorphe et $\left(f_{1}, \ldots, f_{p}, g\right)$ est une suite régulière de fonctions holomorphes. 
Soit $\alpha$ un courant localement résiduel. On associe à $\alpha$ un faisceau $\mathcal{I}_{\alpha}$ de germes de fonctions holomorphes de la manière suivante : en $x \in X$, on pose $\mathcal{I}_{\alpha, x}:=\left\{f \in \mathcal{O}_{x} / f \alpha=0\right\}$. Alors, si l'on écrit explicitement $\alpha$ sur un ouvert $U$ sous la forme :

$$
\sum_{|I|=q}\left(\omega_{I}\right) \wedge\left[1 / f_{p+1}\right] \bar{\partial}\left[1 / f_{1}\right] \wedge \cdots \wedge \bar{\partial}\left[1 / f_{p}\right] d x^{I}
$$

on trouve d'après le lemme 2.7 que le germes d'idéaux $\left\{\mathcal{I}_{\alpha, x}, x \in U\right\}$ sont induits par l'idéal sur $U: \mathcal{I}_{\alpha}(U)=\left\{g \in \mathcal{O}(U) /(\forall I) g \omega_{I} \in\left(f_{1}, \ldots, f_{p}\right)\right\}$. Ainsi, les germes $\mathcal{I}_{\alpha, x}$ définissent un ensemble analytique $S \subset X$, de faisceau définissant $\mathcal{I}_{S}$.

Lemme 2.9. $S$ coïncide avec le support de $\alpha$.

Démonstration. Tout d'abord, supposons que pour l'un des germes de $g \in \mathcal{I}_{\alpha, x}$, $g(x)$ soit non nul ; alors $g$ est inversible, donc $\alpha=1 / g(\alpha g)=0$ au voisinage de $x$, soit $x \notin \operatorname{Supp}(\alpha)$, donc $\operatorname{Supp}(\alpha) \subset S$. Réciproquement, supposons $x \in S$; alors $1 \notin \mathcal{I}_{\alpha, x}$, donc $\alpha$ est non nul au voisinage de $x$, et $x \in \operatorname{Supp}(\alpha)$. On a donc $\operatorname{Supp}(\alpha)=S$.

Lemme 2.10. Supposons $\alpha$ de codimension p. Alors, son support $S$ est de codimension pure $p$.

Démonstration. On a écrit ci-dessus une expression explicite locale pour le faisceau $\mathcal{I}_{\alpha}$ : sur un voisinage ouvert $U$ de $x \in X$, on a : $\mathcal{I}_{\alpha}(U)=\{g \in$ $\left.\mathcal{O}(U) / g \omega_{I} \in\left(f_{1}, \ldots, f_{p}\right)\right\}$. Sur $U$, écrivons une décomposition primaire $\left\{f_{1}=\right.$ $\left.\cdots=f_{p}=0\right\}=I_{1} \cap \cdots \cap I_{k}$, les $I_{j}$ définissant les composantes irréductibles $S_{j}$ (chacune de codimension $p$ ) de $\left\{f_{1}=\cdots=f_{p}=0\right\}$. L'idéal $\mathcal{I}_{\alpha}(U)=$ $\left\{g \in \mathcal{O}(U) /(\forall I) g \omega_{I} \in\left(f_{1}, \ldots, f_{p}\right)\right\}$ s'écrit comme une intersection d'idéaux $\mathcal{I}_{I}:=\left\{g \in \mathcal{O}(U) / g \omega_{I} \in\left(f_{1}, \ldots, f_{p}\right)\right\}$; pour montrer que le support $S \cap U$ de $\mathcal{I}_{\alpha}(U)$ est de codimension pure $p$, il suffit de montrer que le support de chaque $\mathcal{I}_{I}$ est de codimension pure $p$ (car $S \cap U$ est leur réunion). Mais $g \omega_{I} \in\left(f_{1}, \ldots, f_{p}\right)$ équivaut à : $g$ s'annule sur toutes les composantes $S_{j}$ telles que $\omega_{I} \notin I_{j}$. Le support de $\mathcal{I}_{I}$ est donc une réunion d'un nombre fini des $S_{j}, 1 \leq j \leq k$; et il en est de même du support de $\mathcal{I}_{\alpha}(U)$.

Soit $\alpha$ un courant localement résiduel de bidegré $(q, p)$ sur $X$, de support $S$. Supposons que le support $S$ ne soit pas irréductible ; soit $S=S_{1} \cup \cdots \cup S_{k}$ une décomposition irréductible. Alors :

Lemme 2.11. Il existe une décomposition $\alpha=\sum_{i=1}^{k} \alpha_{i}$, avec $\alpha_{i}$ localement résiduel de même bidegré et de support $S_{i}$.

Démonstration. Soit $S_{i}$ l'une des composantes irréductibles. On définit $\alpha_{i}$ comme suit. $\alpha_{i}$ est nul en dehors de $S_{i}$. En un point $x \in S_{i}$, on définit $\alpha_{i}$ sur un voisinage ouvert $U$ de $x$ de la manière suivante. Soit $S \cap U=S_{1}^{\prime} \cap \cdots \cap S_{s}^{\prime}$ une décomposition irréductible de $S \cap U$. On suppose qu'on a choisi la numérotation de sorte que $S_{j} \cap U=\cap_{1 \leq i \leq t} S_{i}^{\prime}$. Alors, pour chaque $j, t+1 \leq j \leq s$, on peut 
trouver une fonction holomorphe $f_{j}$ sur $U$ telle que $f_{j}$ s'annule sur $S_{j}^{\prime}$ sans s'annuler sur aucune $S_{i}^{\prime}, i \leq t$. Alors pour des entiers convenables $i_{1}, \ldots, i_{s-t}$, $g:=f_{t+1}^{i_{1}} \ldots f_{s}^{i_{s-t}}$ ne s'annule sur aucune composante de $S_{j} \cap U$, et $\beta:=g \alpha$ a un support contenu dans $S_{j} \cap U$; alors $1 / g(\beta)$ est bien défini, et coïncide avec $\alpha$ sur $S_{j} \cap U$ en dehors d'une hypersurface de $S_{j} \cap U$; c'est nôtre courant $\alpha_{i}$ sur $U$. On vérifie alors que les différents courants ainsi définis pour deux ouverts $U$ et $V$ coïncident sur $U \cap V$, ce qui nous donne un courant $\alpha_{i}$ global localement résiduel. Alors, on voit que le courant $\sum_{i=1}^{k} \alpha_{i}$ coïncide avec $\alpha$ en dehors d'un sous-ensemble analytique de codimension $>p$, qui est la réunion des intersections $S_{i} \cap S_{j}, i \neq j$. La différence $\alpha-\sum_{i=1}^{k} \alpha_{i}$, à support de codimension $>p$, est donc nulle.

Le courant localement résiduel $\alpha$ vérifie les deux propriétés fondamentales suivantes d'après J.-E. Björk (cf. [1]) :

\section{Lemme 2.12.}

1) $\overline{\mathcal{I}_{S}} \alpha=0$, i.e. pour tout germe en $z \in S$ de fonction holomorphe $f$ au voisinage de z qui s'annule sur $S, \bar{f} \alpha=0$ au voisinage de $z$.

2) a vérifie la propriété d'extension standard définie ci-dessus.

Ces propriétés donnent de plus d'après ([1]) une caractérisation intrinsèque des courants localement résiduels de codimension $p$ :

Lemme 2.13. Soit $\alpha$ un courant sur $X$ de bidegré $(q, p)$, de support $S$. Alors, $\alpha$ est localement résiduel ssi il vérifie les quatre propriétés suivantes:

1) S est de codimension pure $p$;

2) $\bar{\partial} \alpha=0$, en dehors d'un sous-ensemble analytique $T \subset S$ de codimension $p+1$

3) $\overline{\mathcal{I}_{S}} \alpha=0$;

4) $\alpha$ est d'extension standard.

Si $\alpha$ est $\bar{\partial}$-fermé, cette quatrième propriété est superflue.

Pour résumer :

1. Un courant localement résiduel de bidegré $(q, 0)$ est la valeur principale $[\omega]$ associée à une $q$-forme méromorphe $\omega$.

2. Etant donné un courant localement résiduel $\alpha \bar{\partial}$-fermé de bidegré $(q, p)$, il s'écrit localement sous la forme $\omega \wedge \bar{\partial}\left[1 / f_{1}\right] \wedge \cdots \wedge \bar{\partial}\left[1 / f_{p}\right]$, avec $\omega$ une $q$-forme holomorphe. On peut le multiplier par un courant de la forme $[g]$, où $g$ est une forme méromorphe dont le lieu polaire coupe le support de $\alpha$ proprement. Alors, $\bar{\partial}([g] \alpha)=\bar{\partial}[g] \wedge \bar{\partial} \alpha$ est un courant localement résiduel de codimension $p+1$.

3. Soit $\alpha$ un courant localement résiduel de bidegré $(q, p)$. En dehors du support de $\bar{\partial} \alpha$ (qui est un ensemble analytique de codimension $p+1$ et qu'on appellera lieu polaire de $\alpha$ ), $\alpha$ s'écrit localement sous la forme $\omega \wedge \bar{\partial}\left[1 / f_{1}\right] \wedge \cdots \wedge \bar{\partial}\left[1 / f_{p}\right]$, avec $\omega$ une $q$-forme holomorphe. Aux points de 
son lieu polaire, $\alpha$ s'écrit localement sous la forme $\omega \wedge\left[1 / f_{p+1}\right] \bar{\partial}\left[1 / f_{1}\right] \wedge$ $\cdots \wedge \bar{\partial}\left[1 / f_{p}\right]$, avec $\omega$ une $q$-forme holomorphe, et $\left(f_{1}, \ldots, f_{p}, f_{p+1}\right)$ une suite régulière de fonctions holomorphes.

Soit $Y$ un sous-ensemble analytique de $X$ de dimension pure. Soit $\omega$ une $q$ forme méromorphe sur $Y$; on a vu ci-dessus qu'on peut définir le courant valeur principale $[\omega]$ sur $Y$. Alors le courant $\omega \wedge[Y]$, est défini par $\omega \wedge[Y](\phi):=$ $[\omega]\left(i^{*}(\phi)\right)$, avec $i$ l'inclusion $i: Y \subset X$. Alors on a le résultat suivant :

Lemme 2.14. Le courant $\omega \wedge[Y]$ est localement résiduel.

Démonstration. Le résultat est local. Si $Y$ est de codimension 1, c'est un résultat classique : $\omega$ est le résidu de Poincaré-Leray d'une $(q+1)$-forme méromorphe $\Psi$ sur $X$ à pôle logarithmique sur $Y$. Si on écrit $\Psi=\Psi^{\prime} / g$, avec $\{g=0\}$ coupant $Y$ proprement et $\Psi^{\prime}$ holomorphe en dehors de $Y$, on a : $\omega \wedge[Y]=[1 / g] \bar{\partial}\left[\Psi^{\prime}\right]$. Supposons qu'on ait montré le lemme pour $Y$ de codimension $p-1$. Alors, localement, $Y$ est contenu dans un ensemble analytique $Y^{\prime}$ de codimension $p-1$, et le courant $[\omega]$ sur $Y$ définit un courant sur $Y^{\prime}$. On a donc : $\omega \wedge[Y]=[1 / g] \bar{\partial}\left(\Psi^{\prime} \wedge\left[Y^{\prime}\right]\right)$, avec $\Psi^{\prime}$ une certaine forme méromorphe sur $Y^{\prime}$. Mais on sait que $\Psi^{\prime} \wedge\left[Y^{\prime}\right]$ est localement résiduel. $g$ étant une fonction holomorphe telle que $\{g=0\}$ coupe $Y$ proprement, il en est de même de $[1 / g] \bar{\partial}\left(\Psi^{\prime} \wedge\left[Y^{\prime}\right]\right)=\omega \wedge[Y]$.

\subsection{Lien avec le résidu ponctuel de Grothendieck}

Considérons $\alpha$ un courant localement résiduel de bidegré maximal $(n, n)$ (donc $\bar{\partial}$-fermé). Alors, le support de $\alpha$ est discret. Si de plus, il est fini, on peut calculer $\alpha$ sur la fonction constante 1. On obtient alors :

$$
\alpha(1)=\sum_{P \in \operatorname{Supp}(\alpha)} \operatorname{Res}_{P}\left[\begin{array}{c}
\omega \\
f_{1}, \ldots, f_{n}
\end{array}\right],
$$

où $\operatorname{Res}_{P}[\bullet]$ est le résidu ponctuel de Grothendieck, et $\alpha$ s'écrit au point $P$ sous la forme $\omega \wedge \bar{\partial}\left[1 / f_{1}\right] \wedge \cdots \wedge \bar{\partial}\left[1 / f_{p}\right]$, avec $\omega$ un germe de $n$-forme holomorphe en $P$ et $\left(f_{1}, \ldots, f_{n}\right)$ une suite de $n$ germes fonctions holomorphes en $P$ admettant $P$ comme zéro isolé.

Supposons en particulier $X=\mathbb{C}^{n}$, avec des coordonnées $x_{1}, \ldots, x_{n}$. Alors :

Lemme 2.15. Supposons $\alpha(Q)=0$ pour tout polynôme $Q \in \mathbb{C}\left[x_{1}, \ldots, x_{n}\right]$. Alors $\alpha=0$.

Démonstration. Soit $\phi$ une fonction lisse. On peut par interpolation trouver un polynôme $P$ tel que $\phi-P$ s'annule à un ordre suffisant en chaque point du support de $\alpha$, pour que $\alpha(\phi)=\alpha(P)$ d'après le lemme 2.7. Si donc $\alpha(Q)=0$ pour tout polynôme $Q$, on en déduit $\alpha=0$. 


\section{Sur la transformation d'Abel-Radon}

\subsection{Image directe d'un courant par une application holomorphe propre sur son support}

Soit $\phi: X \rightarrow T$ un application holomorphe d'un espace analytique réduit $X$ de dimension pure $m$ vers un espace analytique réduit $T$ de dimension pure $n$. On a un opérateur image inverse sur les formes lisses, tel que $\phi^{*}(\Phi \wedge \Psi)=$ $\phi^{*}(\Phi) \wedge \phi^{*}(\Psi)$, et $\phi^{*}(d \Psi)=d \phi^{*}(\Psi)$. $\phi$ étant holomorphe, $\phi^{*}$ conserve le bidegré, et donc commute avec $\partial$ et $\bar{\partial}$.

Soit $\alpha$ un courant de bidegré $(r, s)$ (donc de bidimension $(m-r, m-s)$ ) sur $X$, tel que la restriction de $\phi$ au support $S:=\operatorname{Supp}(\alpha)$ de $\alpha$ est propre (on dit que $\alpha$ est à support $\phi$-propre). On peut associer à $\alpha$ un courant $\phi_{*}(\alpha)$ ('image directe de $\alpha$ par $\phi$ ) sur $T$, par la formule $\phi_{*}(\alpha)(\psi):=\alpha \wedge \phi^{*}(\psi)(1)$. Le membre de droite est bien défini, car le courant $\alpha \wedge \phi^{*}(\psi)$ est à support compact (contenu dans $S \cap \phi^{-1}(\operatorname{Supp}(\psi))$ ), et définit donc une forme linéaire continue sur les formes lisses (avec la topologie usuelle). On voit immédiatement que la bidimension du courant est inchangée ; donc si $p:=m-n \geq 0, r<p$ ou $s<p$ implique $\phi_{*}(\alpha)=0$. On voit que $\phi_{*}$, pour les courants à support $\phi$-propre, commute avec $d, \partial, \bar{\partial}$; par exemple :

$$
\phi_{*}(\bar{\partial} \alpha)(\psi)=\bar{\partial} \alpha\left(\phi^{*}(\psi)\right)=\alpha\left(\bar{\partial} \phi^{*}(\psi)\right)=\bar{\partial} \phi^{*}(\psi)=\phi^{*}(\bar{\partial} \psi)=\bar{\partial}\left(\phi_{*}(\alpha)\right)(\psi) .
$$

De plus, pour une forme lisse $\Psi$ sur $T$, on a : $\phi_{*}\left(\phi^{*}(\Psi) \wedge \alpha\right)=\Psi \wedge \phi_{*}(\alpha)$. Enfin, on voit que $\operatorname{Supp}\left(\phi_{*}(\alpha)\right) \subset \phi(\operatorname{Supp}(\alpha))$.

Soit maintenant $\alpha$ un courant localement résiduel sur $X$, à support $S \phi$ propre, de bidegré $(r, s) . S$ est un ensemble analytique de codimension pure $s$. Soit $\beta:=\phi_{*}(\alpha)$. Son support est contenu dans $S^{\prime}:=\phi(S)$, qui est d'après le théorème de Remmert ([15]) un sous-ensemble analytique fermé de $T$.

Proposition 3.1. $\beta$ est un courant localement résiduel de bidegré $(r-p, s-p)$ sur $T$. En particulier, si $(r, s)=(q+p, p)$, $\beta$ est une q-forme méromorphe, qui est régulière si $\alpha$ est $\bar{\partial}$-fermé.

Démonstration. Il suffit d'après ce qu'on a vu de faire la démonstration en supposant $S$ irréductible, ce qu'on suppose maintenant. Alors, $S^{\prime}$ est aussi irréductible. Le support de $\beta$ est contenu dans $S^{\prime}$. Supposons d'abord $\alpha \bar{\partial}$ fermé. Alors, $\beta$ est $\bar{\partial}$-fermé, de bidegré $(r-p, s-p)$. Si $\mathcal{I}_{S^{\prime}}$ est le faisceau d'idéaux définissant $S^{\prime}$, on déduit du fait que $\overline{\mathcal{I}}_{S} \alpha=0$, le fait que $\overline{\mathcal{I}}_{S^{\prime}} \beta=0$. Supposons $\operatorname{dim}\left(S^{\prime}\right)<\operatorname{dim}(S)$. On peut alors montrer que $\beta$ est nul. Il suffit, par itération, de la montrer pour les points lisses de $S^{\prime}$. $S^{\prime}$ s'écrit alors sous la forme : $z_{1}=\cdots=z_{r}=0$, pour un système $\left(z_{1}, \ldots, z_{n}\right)$ de coordonnées holomorphes avec $r>s-p$. Du fait que $\overline{\mathcal{I}}_{S^{\prime}} \beta=0$ et $\bar{\partial} \beta=0$, on en déduit $d \overline{z_{i}} \wedge \beta=0(1 \leq i \leq r)$. Mais $\beta$ est de bidegré $(r-p, s-p)$ : les seules formes sur lesquelles il pourrait ne pas s'annuller doivent comporter dans leur décomposition avec les coordonnées $z_{i}$ un $d \overline{z_{i}}$, avec $1 \leq i \leq r$ (sinon il y aurait strictement moins de $n-s+p d \overline{z_{i}}$ ). On voit donc que $\beta=0$. 
Supposons maintenant $\operatorname{dim}(S)=\operatorname{dim}\left(S^{\prime}\right)$. Alors, la caractérisation de J.-E. Björk nous montre que $\beta$ est localement résiduel.

Supposons maintenant que $\alpha$ n'est pas $\bar{\partial}$-fermé, avec $\operatorname{dim}\left(S^{\prime}\right)$ quelconque. Alors, $\bar{\partial} \alpha$ l'est ; son image $\bar{\partial} \beta$ est donc un courant localement résiduel, à support de codimension pure. Si $\operatorname{dim}\left(S^{\prime}\right)<\operatorname{dim}(S)$, on en déduit que $\bar{\partial} \beta$ est un courant localement résiduel de codimension $s-p+1, \beta$ ayant lui-même son support contenu dans un sous-ensemble analytique de codimension $\geq s-p+1$. Cela implique d'après le théorème d'unicité de [4] que $\bar{\partial} \beta=0$. Supposons enfin $\operatorname{dim}(S)=\operatorname{dim}\left(S^{\prime}\right)$. Alors, d'après ce qu'on a déjà vu, $\bar{\partial} \beta=\phi_{*}(\alpha)$ est localement résiduel, de support $T^{\prime}$ de codimension $s-p+1$. De plus, en dehors de $T^{\prime}, \beta$ est localement résiduel. Soit $U$ un ouvert de $T$. On peut encore une fois par linéarité se limiter au cas où le support $S \cap \phi^{-1}(U)$ de $\alpha$ au-dessus de $U$ est irréductible ; alors le support de $\beta S^{\prime} \cap U$ est irréductible dans $U$. Supposons que $g$ est une fonction holomorphe dans $U$, telle que $\{g=0\}$ ne s'annule pas sur $S^{\prime} \cap U$. Alors, on voit que $\left\{\phi^{*}(g)=0\right\}$ ne s'annule pas sur $S \cap \phi^{-1}(U)$, et donc :

$$
\lim _{\epsilon \rightarrow 0} \beta(\chi(|g| / \epsilon) \psi)=\lim _{\epsilon \rightarrow 0} \alpha\left(\chi(|g \circ \phi| / \epsilon) \phi^{*}(\psi)\right)=\alpha\left(\phi^{*}(\psi)\right)=\beta(\psi),
$$

ce qui montre que $\beta$ reste d'extension standard. On voit donc que dans tous les cas, $\beta$ reste localement résiduel.

Supposons en particulier $\alpha$ de bidegré $(q+p, p)$ sur $X$, avec un lieu polaire $Z$. Alors $\beta$ est de la forme $[\omega]$, sur $T$, avec $\omega$ une $q$-forme méromorphe à lieu polaire contenu dans $Z^{\prime}=\phi(Z)$. Si $\alpha$ est $\bar{\partial}$-fermé, $[\omega]$ est $\bar{\partial}$-fermé, et donc $\omega$ est régulière sur $T$.

Remarque. Supposons $m=n$, et $\phi: X \rightarrow T$ propre. Si $\alpha=[\psi]$ pour une forme méromorphe $\psi$ sur $X$, la forme méromorphe $\omega$ telle que $\phi_{*}([\psi])=[\omega]$ s'appelle la trace de $\psi$ pour $\phi$, et se note encore $\phi_{*}(\psi)$. Si $\psi$ est régulière, $\phi_{*}(\psi)$ l'est aussi.

\subsection{Une expression explicite pour l'image directe, dans le cas d'une submersion analytique}

Soit $X$ une variété analytique de dimension $n$, et $T$ un espace analytique réduit irréductible connexe de dimension $m \geq n$. Soit $\phi: T \rightarrow X$ une submersion analytique, c'est-à-dire qu'il existe pour tout point $z \in T$ un voisinage ouvert $W$ de $z$ et un biholomorphisme $\mu_{z}: W \simeq U \times V$, tel que $\phi=\pi \circ \mu_{z}$, avec $\pi$ la projection naturelle $\pi: U \times V \rightarrow U, V \subset \Omega$ un sous-ensemble analytique d'un ouvert $W \subset \mathbb{C}^{p}$ et $U$ un voisinage ouvert de $\phi(z)$ dans $X$. Autrement dit, pour chaque $x \in X$, on peut prendre un système de coordonnées holomorphes $\left(x_{1}, \ldots, x_{n}\right)$ sur un voisinage ouvert $U$ de $x$, tel que en chaque point régulier de $z \in \phi^{-1}(U)$, on peut compléter $\left(x_{1}, \ldots, x_{n}\right)$ en un système de coordonnées holomorphes $\left(x_{1}, \ldots, x_{n}, y_{1}, \ldots, y_{p}\right)$ au voisinage de $z$.

Soit $\Psi$ une forme différentielle de classe $\mathcal{C}^{k}(k \geq 0)$ sur $T$, telle que la restriction de $\phi$ au support de $\Psi$ est propre. $\Psi$ définit donc un courant 
$[\Psi](\Phi):=\int_{T} \Psi \wedge \Phi$ sur $T$, et $\phi$ est propre sur le support de ce courant. On identifie souvent $\Psi$ et le courant $[\Psi]$ associé ; ils ont le même support. On peut donc définir son image directe $\Phi_{*}([\Psi])$.

Lemme 3.2. $\phi_{*}([\Psi])=[\mu]$, pour $\mu$ une forme différentielle de classe $\mathcal{C}^{k}$ sur $X$. En particulier, l'image directe d'une forme lisse à support compact est une forme lisse à support compact.

Démonstration. Une forme $\psi$ sur $T$ est dite verticale si, étant donné une trivialisation locale $\mu: W \rightarrow U \times V$ sur un ouvert $W$ de $T, \mu_{*}(\psi)$ s'écrit sur $U \times V$ sous la forme $\sum_{I} \psi_{I} \rho_{I}$, avec $\psi_{I}$ des fonctions sur $U \times V$, et $\rho_{I}$ des formes sur $V$. En particulier, les indices de bidegré d'une telle forme sont inférieurs à $p$.

Supposons d'abord que $T:=X \times F$, avec $F$ un espace analytique réduit connexe de dimension $p$, et $\phi: T \rightarrow X$ la projection standard. Soit $\psi$ une forme sur $T$ de bidegré $(r, s)$ arbitraire. On se place au-dessus d'un ouvert $U$ de $X$, avec des coordonnées holomorphes $x_{1}, \ldots, x_{n}$. $\psi$ s'écrit de manière unique au dessus de $U$ sous la forme : $\psi=\sum_{I, J \subset\{1, \ldots, n\}} \psi_{I, J} \wedge d x^{I} \wedge d \bar{x}^{J}$, où les $\psi_{I, J}$ sont des formes verticales. Comme les indices de bidegré de $\psi_{I, J}$ sont inférieurs à $p$, les multi-indices $I$ et $J$ intervenant dans la somme sont nécessairement de longueur respectivement supérieure à $r^{\prime}:=r-p$ et $s^{\prime}:=s-p$. Si $r<p$, ou $s<p$, on a vu que $\phi_{*}([\psi])=0$. Supposons $r, s \geq p$.

$$
\psi=\sum_{I=\left(i_{1}, \ldots, i_{r^{\prime}}\right), i_{1}<\cdots<i_{r^{\prime}}, J=\left(j_{1}, \ldots, j_{s^{\prime}}\right), j_{1}<\cdots<j_{s^{\prime}}} \psi_{I, J} d x^{I} \wedge d \bar{x}^{J}+\psi^{\prime},
$$

où $\psi^{\prime}$ regroupe les termes correspondant à $\operatorname{card}(I)>r^{\prime}$ ou $\operatorname{card}(J)>s^{\prime}$. On a vu que $\phi_{*}\left(\left[\psi^{\prime}\right]\right)=0$. On en déduit :

$$
\phi_{*}(\psi)=\sum_{I, J, \operatorname{card}(I)=r^{\prime}, \operatorname{card}(J)=s^{\prime}} \phi_{*}\left(\psi_{I, J}\right) d x^{I} \wedge d \bar{x}^{J},
$$

où $\psi_{I, J}$ sont des formes verticales de bidegré $(p, p)$.

Il reste donc à calculer $\phi_{*}(\psi)$, pour $\psi$ verticale de bidegré $(p, p)$. Soit dans ce cas $\psi_{x}$ la forme sur $F$ obtenue pour une valeur $x$ fixée. Alors, d'après le théorème de Fubini, $\mu(x):=\int_{F} \psi_{x}$ est localement intégrable et pour $f$ lisse à support compact :

$$
\int_{U \times F} \psi \wedge f(x) d x \wedge d \bar{x}=\int_{U} f(x) d x \wedge d \bar{x} \int_{F} \psi_{x} .
$$

Dans le cas général où $\psi$ est localement intégrable, on voit donc que le courant $\phi_{*}(\psi)$ est représenté par la fonction localement intégrable $\mu(x):=\int_{F} \psi_{x}$. Si $\psi$ est de classe $\mathcal{C}^{k}$ à support compact, on sait, par exemple d'après L. Schwartz ([17]) que $\mu(x):=\int_{F} \psi_{x}$ est de classe $\mathcal{C}^{k}$.

On se place maintenant dans le cas général d'une submersion analytique $\phi: T \rightarrow X$. Soit un recouvrement d'ouverts relativement compacts $\left(U_{i}\right)_{i \in I}$ de $T$ 
par des ouverts sur lesquels $\phi$ est trivialisable. On se donne une partition de l'unité associée $\left(\chi_{i}\right)_{i \in I}: \sum_{i \in I} \chi_{i}=1$. Soit $\psi$ une forme $\mathcal{C}^{k}$ sur $T, \phi$-propre. On a : $\psi=\sum_{i \in I} \psi \chi_{i}$, et donc $\phi_{*}(\psi)=\sum_{i \in I} \phi_{*}\left(\chi_{i} \psi\right)$. La somme est bien définie, car pour tout point $x \in T$, il existe un voisinage $U_{x}$ relativement compact, tel que $\phi^{-1}\left(\overline{U_{x}}\right) \cap \operatorname{supp}(\psi)$ est compact, donc au-dessus de $U_{x}, \sum_{i \in I} \psi \chi_{i}$ est égale à une somme finie $\sum_{i \in J} \psi \chi_{i}$, et sur $U_{x}$ l'image directe est la somme des images directes. Chaque terme de la somme est $\mathcal{C}^{k}$, d'après ce qu'on a vu pour les projections ; donc la somme est de classe $\mathcal{C}^{k}$.

Soit maintenant $\alpha$ un courant localement résiduel de bidegré $(q, p)$ sur $T$, $\bar{\partial}$-fermé, tel que la restriction de $\phi$ au support $S$ de $\alpha$ est propre. Soit $x_{1}, \ldots, x_{n}$ un système de coordonnées holomorphes dans un ouvert $U$ de $X$. On peut écrire au-dessus de $U: \alpha=\sum_{I|I|=q} \alpha_{I} d x^{I}$, avec $\alpha_{I}$ un courant de bidegré $(q-|I|, p)$, qui lorsqu'on l'écrit dans un système de coordonnées locales contenant les $x_{1}, \ldots, x_{n}$, ne contient pas les $d x_{i}$. Alors sur $U$ on a $\phi_{*}(\alpha)=\sum_{I, I|I|=q-p} \mu_{I}(x) d x^{I}$, avec $d x^{I}:=d x_{i_{1}} \wedge \cdots \wedge d x_{i_{q-p}}$, et $\mu_{I}$ une fonction holomorphe sur $U$ (on remarque que si $|I|<q-p, \phi_{*}\left(\alpha_{I}\right)=0$. Supposons que pour tout $x \in U$, la fibre $F_{x}=\phi^{-1}(x)$ coupe $S$ en un nombre fini de points. On va donner une expression explicite pour $\mu:=\phi_{*}(\alpha)$; pour cela, il suffit d'après ce qui précède de faire le calcul pour $\alpha$ de bidegré $(p, p)$, ne contenant pas les $d x_{i}$.

Lemme 3.3. Soit $\alpha$ un courant localement résiduel de bidegré ( $p, p)$. Alors

$$
\phi_{*}(\alpha)=\sum_{Q \in F_{x} \cap S} \operatorname{Res}_{Q}\left[\begin{array}{c}
\omega \\
f_{1}, \ldots, f_{p}
\end{array}\right],
$$

où on a écrit dans un voisinage de $Q: \alpha=\omega \wedge \bar{\partial}\left[1 / f_{1}\right] \wedge \cdots \wedge \bar{\partial}\left[1 / f_{p}\right]$, avec $\omega$ une p-forme holomorphe.

Démonstration. On a $\phi_{*}(\alpha)=\phi_{*}\left(\alpha^{\prime}\right)$ où $\alpha^{\prime}$ est la composante de la décomposition $\alpha=\sum_{I} \alpha_{I} d x^{I}$ ne contenant pas de $d x_{i}$. Par conséquent, on peut supposer que $\alpha$ ne contient pas de $d x_{i}$. Donc, $\alpha$ s'écrit au voisinage d'un point $Q \in F_{x}$ sous la forme : $\omega \wedge \bar{\partial}\left[1 / f_{1}\right] \wedge \cdots \wedge \bar{\partial}\left[1 / f_{p}\right]$, où $\omega$ est une $p$-forme holomorphe au voisinage de $Q$, ne contenant pas de $d x_{i}$. Mais comme $S$ et $F_{x}$ se coupent en un nombre fini de points, l'expression $\omega \wedge \bar{\partial}\left[1 / f_{1}\right] \wedge \cdots \wedge \bar{\partial}\left[1 / f_{p}\right]$ définit par restriction sur $F_{x}$ au voisinage de $Q \in F_{x}$ un courant localement résiduel à support fini $\beta_{x}$ sur la fibre $F_{x}$. Il suffit alors de montrer que si $\alpha_{Q}$ est le germe de courant dont le support rencontre $Q, \phi_{*}\left(\alpha_{Q}\right)$ vaut $\operatorname{Res}_{Q}\left[\begin{array}{c}\omega \\ f_{1}, \ldots, f_{p}\end{array}\right]$. Cela se fait en application les formules intégrales données ci-dessus pour les résidus, par une application du théorème de Fubini suivie d'un passage à la limite.

\subsection{Image inverse d'un courant par une submersion analytique}

Soit $\phi: T \rightarrow X$ une submersion analytique comme ci-dessus. L'image inverse d'un courant $\beta$ sur $X$ par $\phi$ est définie par : $\phi^{*}(\beta)(\psi):=\beta\left(\phi_{*}(\psi)\right)$. Le bidegré reste inchangé. On vérifie de plus : $\operatorname{Supp}\left(\phi^{*}(\beta)\right) \subset \phi^{-1}(\operatorname{Supp}(\beta))$, et $\phi^{*}(\beta \wedge \psi)=\phi^{*}(\beta) \wedge \phi^{*}(\psi)$ pour une forme lisse $\psi$. 
Proposition 3.4. Si $\beta$ est un courant résiduel sur $X, \alpha:=\phi^{*}(\beta)$ est résiduel sur $T$. De plus, le résidu commute avec l'image inverse : si $Y_{1}, \ldots, Y_{k}$ sont des hypersurfaces se coupant proprement sur $X$, de sorte que $\beta=\operatorname{Res}_{Y_{1}, \ldots, Y_{k}} \Psi$ avec une q-forme méromorphe dont le lieu polaire coupe $Y_{1} \cap \cdots \cap Y_{k}$ proprement, alors

$$
\alpha=\operatorname{Res}_{Y_{1}^{\prime}, \ldots, Y_{k}^{\prime}} \Psi^{\prime},\left(\Psi^{\prime}=\phi^{*}(\Psi), Y_{i}^{\prime}=\phi^{-1}\left(Y_{i}\right)(1 \leq i \leq k)\right) .
$$

De plus, soit $Z$ un sous-ensemble analytique de $X$, et $\omega$ une forme méromorphe sur Z. Alors on $a$ :

$$
\phi^{*}(\omega \wedge[Z])=\phi^{*}(\omega) \wedge\left[\phi^{-1}(Z)\right] .
$$

Démonstration. Plaçons-nous sur un ouvert $U$ de $X$, où $\alpha$ s'écrit sous la forme $\omega\left[1 / f_{k+1}\right] \wedge \bar{\partial}\left[1 / f_{1}\right] \wedge \cdots \wedge \bar{\partial}\left[1 / f_{k}\right]$, avec $\left(f_{1}, \ldots, f_{k+1}\right)$ une suite régulière de fonctions holomorphes. Posons $g_{i}:=\phi^{*}\left(f_{i}\right)(1 \leq i \leq k+1)$. $\left(g_{1}, \ldots, g_{k+1}\right)$ forment toujours une suite régulière sur $\phi^{-1}(U)$, puisque la codimension de $\left\{g_{1}=\cdots=g_{p+1}=0\right\}$ est la même que celle de $\left\{f_{1}=\cdots=f_{k+1}=0\right\}$. On vérifie facilement sur $\phi^{-1}(U)$ l'égalité des deux courants

$$
\phi^{*}\left(\left[1 / f_{k+1}\right] \wedge \bar{\partial}\left[1 / f_{1}\right] \wedge \cdots \wedge \bar{\partial}\left[1 / f_{k}\right]\right)
$$

et

$$
\left.\left[1 / g_{k+1}\right)\right] \bar{\partial}\left[1 / g_{1}\right] \wedge \cdots \wedge \bar{\partial}\left[1 / g_{k}\right] .
$$

L'égalité de ces deux courants revient à l'égalité, pour $\psi$ une forme lisse à support compact sur $T$ de bidegré $(m, m-k)$, des deux intégrales :

$$
\int_{T}\left[1 / g_{k+1}\right] \bar{\partial}\left[1 / g_{1}\right] \wedge \cdots \wedge \bar{\partial}\left[1 / g_{k}\right] \wedge \psi
$$

et

$$
\int_{X}\left[1 / f_{k+1}\right] \bar{\partial}\left[1 / f_{1}\right] \wedge \cdots \wedge \bar{\partial}\left[1 / f_{k}\right] \wedge \phi_{*}(\psi) .
$$

L'égalité de ces deux intégales se montre par la formule de Fubini généralisée, pour $\psi$ à support dans un ouvert où $\phi$ est trivialisable ; dans le cas général, on utilise une partition de l'unité. En particulier, supposons qu'on parte d'un courant résiduel de la forme $\alpha=\omega \wedge[Y]=\omega \wedge d f_{1} \wedge \cdots \wedge d f_{k} \wedge \bar{\partial}\left[1 / f_{1}\right] \wedge \cdots \wedge$ $\bar{\partial}\left[1 / f_{k}\right]$ sur un ouvert $U$ de $X$; alors $\phi^{*}(\alpha)$ est de la forme : $\phi^{*}(\omega) \wedge d g_{1} \wedge$ $\cdots \wedge d g_{k} \wedge \bar{\partial}\left[1 / g_{1}\right] \wedge \cdots \wedge \bar{\partial}\left[1 / g_{k}\right]=\phi^{*}(\omega) \wedge\left[\phi^{-1}(Y)\right] \operatorname{sur} \phi^{-1}(U)$.

\subsection{La transformation d'Abel-Radon}

On se donne les objets suivants :

1. Un espace analytique réduit $X$ de dimension pure $N$.

2. Un espace de paramètres $T$, espace analytique réduit connexe.

3. Un espace d'incidence $I \subset T \times X$, sous-ensemble analytique de dimension pure, avec les projections naturelles $p_{1}: I \rightarrow T$ et $p_{2}: I \rightarrow X$ et les propriétés suivantes :

1) La projection $p_{1}: I \rightarrow T$ est propre.

2) La projection $p_{2}: I \rightarrow X$ est une submersion. 
On a vu ci-dessus qu'on peut définir l'image inverse d'un courant par une submersion, ainsi que l'image directe d'un courant par une application propre ; on définit la transformation $\mathcal{R}$, qu'on nomme transformation d'Abel-Radon par la formule ci-dessous, qui associe à un courant $\alpha$ de bidegré $(r, s)$ sur $X$ un courant de bidegré $(r-p, s-p)$ sur $T(\operatorname{avec} p=\operatorname{dim}(I)-\operatorname{dim}(T))$ :

Définition 3.5.

$$
\mathcal{R}(\alpha)=p_{1 *}\left(p_{2}{ }^{*}(\alpha)\right) .
$$

Soit $Y$ un sous-ensemble analytique de $X$ de codimension pure $p$. Alors, posons $I_{Y}:=p_{2}^{-1}(Y) \subset I$. $I_{Y}$ définit une famille de zéro-cycles sur $Y$, avec les deux projections standard $\pi_{1}: I_{Y} \rightarrow T$ et $\pi_{2}: I_{Y} \rightarrow Y$. On peut définir la transformation d'Abel d'une forme méromorphe $\omega$ sur $Y$, par $\mathcal{A}(\omega):=\pi_{1 *}\left(\pi_{2}^{*}(\omega)\right)$, qui est une forme méromorphe sur $T$ (cf. [6]). Alors :

Proposition 3.6. $\mathcal{A}(\omega)=\mathcal{R}(\omega \wedge[Y])$.

Démonstration. On a $p_{2}{ }^{*}(\omega \wedge[Y])=\pi_{2}^{*}(\omega) \wedge\left[I_{Y}\right]$. De plus, $p_{1 *}\left(\psi \wedge\left[I_{Y}\right]\right)=$ $\pi_{1 *}(\psi)$, avec $\psi:=\pi_{2}^{*}(\omega)$.

\subsection{Démonstration du théorème 1.1}

On a vu ci-dessus que l'image inverse d'un courant localement résiduel par une submersion analytique, de même que son image directe par une application holomorphe propre, reste un courant localement résiduel. La transformation d'Abel-Radon, qui est la succession d'une submersion est d'une application propre, laisse donc stable la famille des courants localement résiduels. De plus, on a déjà vu que les opérateurs $d, \bar{\partial}, \partial$ sur les courants commutent avec l'image directe (resp. l'image inverse) par une application holomorphe propre (resp. submersive). $\mathcal{R}$ commute donc avec les opérateurs $d, \bar{\partial}, \partial$; si $\alpha$ est un courant $\bar{\partial}$-fermé, $\mathcal{R}(\alpha)$ l'est aussi.

\section{Rappels de quelques résultats de prolongement analytique}

On va rappeler dans cette courte section quelques résultats de prolongement analytique qui vont nous servir par la suite.

Le lemme suivant est démontré dans Rothstein, [16] :

Lemme 4.1. Soit $D \subset \mathbb{C}^{n}$, et $\Delta_{1} \subset \Delta_{2} \subset \mathbb{C}^{m}$ deux polydisques. Soit $f(a, b)$ : $D \times \Delta_{1} \rightarrow \mathbb{C}$ une fonction holomorphe. On suppose que pour $a \in D$ fixé, $f$ s'étend holomorphiquement dans $D \times \Delta_{2}$. Alors, $f$ s'étend holomorphiquement dans $D \times \Delta_{2}$.

Le lemme suivant a été montré pour $N=2$ par W. Kneser ([13]) ; la démonstration s'adapte pour $N$ plus grand : 
Lemme 4.2. Soit $f$ une fonction méromorphe sur $\Delta^{N}$, où $\Delta$ est le disque $\{z \in$ $\mathbb{C},|z|<1\}$. Alors pour $\left(z_{1}^{0}, \ldots, z_{i-1}^{0}, z_{i+1}^{0}, \ldots, z_{N}^{0}\right)$ fixés dans un ouvert Zariskidense de $\Delta^{N-1}$, on peut considérer la fonction de la variable $z_{i}$

$$
f_{i}\left(z_{i}\right):=f\left(z_{1}^{0}, \ldots, z_{i-1}^{0}, z_{i}, z_{i+1}^{0}, \ldots, z_{N}^{0}\right)
$$

qui est méromorphe sur $\Delta$. On suppose $f_{i}$ rationnelle lorsqu'elle est définie, pour chaque $i, 1 \leq i \leq N$. Alors $f$ est rationnelle.

Lemme 4.3. Soit $U$ un domaine de $\mathbb{P}^{N}$. Si $U$ contient une droite complexe, alors toute q-forme méromorphe $\psi$ définie sur $U$ s'étend en une q-forme rationnelle sur $\mathbb{P}^{N}$.

Démonstration. On se ramène aisément au cas d'une fonction méromorphe, en écrivant $\psi$ comme combinaison, à coefficients méromorphes, de formes rationnelles élémentaires $d x_{i_{1}} \wedge \cdots \wedge d x_{i_{q}}$, avec $x_{1}, \ldots, x_{N}$ un choix convenable de coordonnées affines. Soit un point $P$ sur la droite complexe $\Delta$ dont on suppose l'existence, et un hyperplan $H$ ne contenant pas $P$. Choisir un système de coordonnées affines ayant $P$ comme origine et $H$ comme hyperplan à l'infini revient à se donner $N$ points dans $H$; à savoir, les $N$ points $P_{i}(1 \leq i \leq$ $N)$, intersection de $H$ avec les droites $x_{1}=C s t, \ldots, x_{i-1}=C s t, x_{i+1}=$ $C s t, \ldots, x_{N}=C s t$. Choisissons ces $N$ points dans $H \cap U$, et de sorte que les $N$ droites

$$
x_{1}=0, \ldots, x_{i-1}=0, x_{i+1}=0, \ldots, x_{N}=0
$$

soient contenues dans $U$ (il suffit de les choisir suffisamment proches de $\Delta \cap H$ ). Alors, $f$ est une fonction méromorphe de $x_{1}, \ldots, x_{N}$ dans un voisinage de l'origine, et elle est rationnelle sur toutes les droites $x_{1}=x_{1}^{0}, \ldots, x_{i-1}=$ $x_{i-1}^{0}, x_{i+1}=x_{i+1}^{0}, \ldots, x_{N}=x_{N}^{0}$, pour tout $\left(x_{1}^{0}, \ldots, x_{i-1}^{0}, x_{i+1}^{0}, \ldots, x_{N}^{0}\right)$ dans un ouvert de Zariski dense d'un voisinage ouvert de $0 \in \mathbb{C}^{N+1}$, donc rationnelle. $f$ s'exprime donc rationnellement en fonction des coordonnées affines $\left(x_{1}, \ldots, x_{N}\right)$.

Le lemme précédent découle en fait d'un théorème de prolongement basée sur une notion de pseudoconcavité donnée par A. Andreotti :

Définition 4.4. Soit $X$ une variété analytique connexe. Un domaine $U$ de $X$ est pseudoconcave au sens d'Andreotti, si pour tout point de la frontière $x \in \partial U$, pour tout voisinage ouvert $U_{x}$ de $x$ dans $X$, toute fonction holomorphe sur $U_{x} \backslash \bar{U}$ est holomorphe dans un voisinage de $x$. X est dite pseudoconcave au sens d'Andreotti si elle contient un domaine pseudoconcave au sens d'Andreotti.

Il suffit, d'après un théorème d'Hartogs, pour vérifier que le domaine $U$ est pseudoconcave au sens d'Andreotti, d'exhiber pour tout $x \in \partial U$, un disque analytique $D$ de $X$, tel que $D$ ne rencontre le complémentaire de $U$ qu'en $x$.

Alors, P. Dingoyan a montré dans [5] le théorème suivant :

Lemme 4.5. Soit $U$ un domaine pseudoconcave au sens d'Andreotti d'une variété algébrique complexe $X$. Alors, toute fonction méromorphe sur $X$ est rationnelle. 


\section{Sur les traces des courants localement résiduels}

On se place dans le cas particulier suivant de la transformation d'Abel-Radon : $T$ est une variété analytique de dimension $n, X$ est le produit $T \times \mathbb{P}^{1}$, et $\phi$ est la projection standard $\phi: T \times \mathbb{P}^{1} \rightarrow T$. On note pour $x \in T, F_{x}:=\phi^{-1}(x)$. On se donne sur $X$ un courant $\alpha$, localement résiduel de bidegré $(q, 1)$, et sans composante verticale. On suppose de plus que le support $S$ de $\alpha$, qui est une hypersurface analytique de $X$, ne contient pas $T_{\infty}:=T \times\{\infty\}$. Soit $y$ une coordonnée affine sur $\mathbb{P}^{1}$. Considérons le corps $K$ des fonctions méromorphes sur un ouvert connexe $U$ de $X$. On peut multiplier le courant localement résiduel $\alpha$, par n'importe quelle fonction méromorphe $Q \in K[y]$, puisque le lieu polaire de $Q$ coupe le support de $\alpha$ proprement. L'ensemble des $Q$ tels que $Q \alpha=0$ forme un idéal dans l'anneau principal $K[y]$. Cet idéal est non nul, puisque si $Q(y)=\Pi_{z \in F_{x} \cap S}(y-y(z))=0$ est l'équation de $S$, avec $Q$ un polynôme unitaire de $K[y]$, on a nécessairement $Q^{s} \alpha=0$ pour un certain entier $s$. Cet idéal est donc engendré par un unique polynôme unitaire $P(y)=y^{d}+a_{1} y^{d-1}+\cdots+a_{d}$, avec $a_{i} \in K(1 \leq i \leq d)$.

Remarque. L'ensemble des points $x \in T$ où la fibre $F_{x}$ rencontre $S \cap T_{\infty}$, coïncide en fait avec l'une des fonction méromorphe $a_{i}(1 \leq i \leq d)$ n'est pas holomorphe. En effet, soit $T^{\prime} \subset T$ l'ouvert de Zariski sur lequel toutes les $a_{i}$ sont holomorphes. Au-dessus de $T^{\prime}$, on a $S \subset\{P=0\}$, et donc $F_{x} \cap S$ ne contient aucun point à l'infini. Réciproquement, supposons que l'un des coefficients $a_{i}$ n'est pas holomorphe ; alors on voit qu'en se rapprochant de ce point, l'une des racines $y$ tend vers l'infini.

On définit pour tout $k \in \mathbb{N}$, les "traces" $v_{k}:=\phi_{*}\left(\alpha y^{k}\right)$, et $w_{k}:=\phi_{*}\left(\alpha y^{k} \wedge\right.$ $d y$ ), qui sont des formes méromorphes sur $T$ de degrés respectifs $q-1$ et $q$.

On se place sur un ouvert $U$ de $X$ avec des coordonnées holomorphes $x_{1}, \ldots, x_{n}$. On pose pour tout $k \in \mathbb{N}, v_{k}=\sum_{I,|I|=q-1} v_{k, I} d x^{I}$; de même $w_{k}=\sum_{I,|I|=q} w_{k, I} d x^{I}$.

En prenant l'image directe $\operatorname{par} \phi$ de $y^{k} P(x, y) \alpha=0$, puis de $d y \wedge$ $y^{k} P(x, y) \alpha=0$, on obtient sur $T$ :

\section{Lemme 5.1.}

$$
\begin{gathered}
(\forall k \in \mathbb{N}) v_{k+d}+a_{1} v_{k+d-1}+\cdots+a_{d} v_{k}=0, \\
(\forall k \in \mathbb{N}) w_{k+d}+a_{1} w_{k+d-1}+\cdots+a_{d} w_{k}=0 .
\end{gathered}
$$

On a donc $(\forall k \in \mathbb{N}) v_{k+d, I}+a_{1} v_{k+d-1, I}+\cdots+a_{d} v_{k, I}=0, w_{k+d, I}+a_{1} w_{k+d-1, I}+$ $\cdots+a_{d} w_{k, I}=0$.

Lemme 5.2. Supposons que pour tout $k, 0 \leq k \leq d-1$, on ait $v_{k}=w_{k}=0$ sur U. Alors, $\alpha=0$ sur X. 
Démonstration. Remarquons que $v_{k}$ et $w_{k}$ sont méromorphes ; si donc il sont nuls sur $U$, il le sont sur $T$ (qui est supposé connexe). Tout d'abord, les équations $(\forall k \in \mathbb{N}) v_{k+d}+a_{1} v_{k+d-1}+\cdots+a_{d} v_{k}=0$ nous donnent $(\forall k \geq 0) v_{k}=$ 0 ; de même $(\forall k \geq 0) w_{k}=0$. On peut supposer que au-dessus de $U, S$ ne rencontre pas l'infini $T_{\infty}$, et que $U$ est de Stein. Alors, on peut écrire $\alpha$ sur $U \times \mathbb{C}$ comme un résidu global : $\alpha=\operatorname{Res}_{S} \Psi$, avec $\Psi$ une $q$-forme méromorphe sur $U \times \mathbb{C}\left(S\right.$ ne rencontrant pas $\left.T_{\infty}\right)$. Ecrivons $\Psi$ sous la forme $\Psi:=\psi^{\prime}+\psi \wedge d y$, avec $\psi$ et $\psi^{\prime}$ des formes méromorphes ne contenant pas de $d y$. De même, posons $\psi^{\prime}:=\sum_{I,|I|=q} \psi_{I}^{\prime} d x_{I}$ et $\psi:=\sum_{I,|I|=q-1} \psi_{I} d x_{I}$, où $\psi_{I}, \psi_{I}^{\prime}$ sont des fonctions méromorphes sur $U \times \mathbb{C}$. Soit $I$ fixé, avec $|I|=q-1$. En multipliant par $y^{k}$ et en prenant l'image directe par $\phi$, nous donne, par l'expression explicite de la trace, pour tout $k \geq 0: v_{k, I}=\operatorname{Res}_{F_{x} \cap S} \psi_{I} d y\left(y^{k}\right)$. Mais comme $\phi_{*}\left(\alpha y^{k}\right)=0, v_{k, I}=0$. Le courant sur $F_{x}: \operatorname{Res}_{F_{x} \cap S} \psi_{I} d y$ (à support fini dans $\mathbb{C}$ ) s'annule sur tout polynôme, donc d'après ce qu'on a vu est un courant nul. Donc, $\psi_{I}$ n'a pas de pôle sur $F_{x} \cap S$. Autrement dit, pour $x \in U$ fixé, $\psi_{I}$ se prolonge holomorphiquement à travers $S$. D'après le lemme de prolongement de Rothstein ci-dessus, $\psi_{I}$ se prolonge en fait holomorphiquement sur $U \times \mathbb{C}$.

On montrerait de même, en prenant les traces $\phi_{*}\left(\alpha y^{k} \wedge d y\right)$, que $\psi_{I}^{\prime}$ se prolonge holomorphiquement sur $U \times \mathbb{C}$, pour tout $I,|I|=q$. On en déduit finalement que $\Psi$ se prolonge holomorphiquement à travers $S$ sur $U \times \mathbb{C}$. Par conséquent, $\alpha=\operatorname{Res}_{S} \Psi=0$.

Dans ce qui suit, on suppose pour l'instant $q=n+1$, i.e. on suppose que $\alpha$ est un courant localement résiduel de bidegré $(n+1,1)$. Alors, il n'y a qu'un seul multiindice $I$ de longueur $q$ ou $q-1$ possible pour $d x^{I}$, soit $I=(1, \ldots, n)$. On pose alors : $u_{k}:=v_{I, k}$. Considérons le système carré $d \times d$ d'équations linéaires, à coefficients dans le corps $K$ des fonctions méromorphes sur $U$,

$$
b_{1} u_{k+d-1}+\cdots+b_{d} u_{k}=-u_{k+d}(k=0,1, \ldots, d-1)
$$

avec les inconnues $b_{1}, \ldots, b_{d}$.

Lemme 5.3. Ce système admet $\left(a_{1}, \ldots, a_{d}\right)$ comme unique solution. Par conséquent, le déterminant $M=\left[u_{d-1+i-j}\right]_{0 \leq i, j \leq d-1}$ est non identiquement nul sur $U$.

Démonstration. Tout d'abord, le système admet une solution, soit $\left(a_{1}, \ldots, a_{d}\right)$. Montrons l'unicité : soit $\left(b_{1}, \ldots, b_{d}\right)$ une solution arbitraire : $b_{1} u_{d-1+k}+\cdots+$ $b_{d} u_{k}=0$, pour $k=0, \ldots, d-1$. Mais alors, les équations $u_{k+d}+a_{1} u_{k+d-1}+$ $\cdots+a_{d} u_{k}=0$, sur $U$ pour tout $k \in \mathbb{N}$, impliquent que $b_{1} u_{d-1+k}+\cdots+b_{d} u_{k}=0$ reste valide pour $k \geq d$. On en déduit alors :

$$
(\forall k \in \mathbb{N}) \phi_{*}\left(\left(y^{d}+b_{1} y^{d-1}+\cdots+b_{d}\right) y^{k} \alpha\right)=0,
$$

d'où $\left(y^{d}+b_{1} y^{d-1}+\cdots+b_{d}\right) \alpha=0$ d'après le lemme précédent. Par unicité du générateur unitaire, on en déduit $b_{i}=a_{i}(1 \leq i \leq d)$.

Remarque. Dans le cas où le support de $\alpha$ est "réduit", on peut calculer le déterminant de la manière suivante. Supposons $\alpha:=\omega \wedge[S]$, où $S$ est le support 
de $\alpha$ et $\omega$ une $n$-forme méromorphe sur $S$. On peut écrire $\omega_{i}=f_{i}(x) d x$, sur les différentes composantes $S_{i}$ de $S$ au-dessus de $U$. Alors, le déterminant s'écrit sur $U$ sous la forme :

$$
\Pi_{i<j}\left(y_{i}(x)-y_{j}(x)\right)^{2} \Pi_{i=1}^{d} f_{i}(x) .
$$

Ce qui précède montre que l'on peut retrouver les coefficients $a_{i}$ de $P$ à partir des traces $u_{k}, 0 \leq k \leq 2 d-1$. Le lemme suivant nous permet de retrouver $\alpha$ à partir de ses traces $u_{k}, 0 \leq k \leq 2 d-1$. Tout d'abord, on définit sur $U$ un polynôme $P^{\prime}$ à coefficients holomorphes, de la manière suivante. On pose $a_{i}=p_{i} / q_{i}$ une représentation irréductible en quotient de deux fonctions holomorphes sur $U$; alors, on multiplie $P=y^{d}+a_{1} y^{d-1}+\cdots+a_{d}$ par le plus petit multiple commun $b_{0}$ de $q_{i}, 1 \leq i \leq d$. On obtient ainsi un polynôme $P^{\prime}=b_{0} y^{d}+\cdots+b_{d}$, dont les coefficients sont holomorphes sur $U$, et n'ont pas de commun diviseur. De plus, $S$ a pour équation $P^{\prime}=0$.

Lemme 5.4. On a sur $U \times \mathbb{P}^{1}: \alpha=\operatorname{Res}_{S} r(x, y) d x \wedge d y / P^{\prime}$, avec :

$r(x, y):=y^{d-1} u_{0}+y^{d-2}\left(b_{0} u_{1}+b_{1} u_{0}\right)+\cdots+\left(b_{0} u_{d-1}+b_{1} u_{d-2}+\cdots+b_{d-1} u_{0}\right)$.

Démonstration. Posons $\beta:=\operatorname{Res}_{S} r(x, y) d x \wedge d y / P^{\prime}$, courant résiduel de bidegré $(n+1,1)$ sur $U \times \mathbb{P}^{1}$ sans composante verticale. D'après ce qui précède, il suffit pour montrer $\alpha=\beta$ de montrer que $\phi_{*}\left(\alpha y^{k}\right)=\phi_{*}\left(\beta y^{k}\right)$ pour $k \in \mathbb{N}$. D'après l'expression explicite de la trace, on a : $\phi_{*}\left(\beta y^{k}\right)=\sum_{Q \in F_{x} \cap S} \operatorname{Res}_{Q}\left[\begin{array}{c}r y^{k} d y \\ P^{\prime}\end{array}\right] d x$. La forme méromorphe $y^{k} r / P^{\prime} d y$ est en fait rationnelle en $y$, puisque $P^{\prime}$ et $r$ sont des polynômes en $y$. Plaçons-nous au voisinage d'un point $x \in U$ où les $u_{k}$ sont holomorphes. Alors, $y^{k} r / P^{\prime} d y$ est holomorphe en dehors de l'infini et des points de $F_{x} \cap S$; par conséquent, comme on sait que la somme de ses résidus sur $\mathbb{P}^{1}$ est nulle, il suffit pour calculer la somme de ses résidus aux points de $F_{x} \cap S$ de calculer son résidu au point à l'infini. Si l'on fait le changement de variable $y=1 / y^{\prime}$, on vérifie que ce résidu est précisement $u_{k}$, ce qui montre bien que $\alpha$ et $\beta$ ont les même traces. Ce qui précède montre alors que $\alpha=\beta$ au-dessus des points $x$ où les $u_{k}$ sont holomorphes. Alors $\alpha-\beta$ est localement résiduel de bidegré $(n+1,1)$, à support dans $T_{\infty} \cap S$, qui est de codimension 2 ; il est donc nul.

Corollaire 5.5. Si les $u_{k}(0 \leq k \leq d-1)$ sont holomorphes sur $U$, $\alpha$ est $\bar{\partial}$-fermé au-dessus de $U$.

\subsection{Démonstration du théorème 1.2}

Soit maintenant $\alpha$ localement résiduel de bidegré $(q, 1)$ sur $X \times \mathbb{P}^{1}$. Supposons tout d'abord $\alpha \bar{\partial}$-fermé sur $U \times \mathbb{P}^{1}$, et que les $v_{k}, w_{k}, k \in \mathbb{N}$ se prolongent holomorphiquement sur $T$. Le lemme de Zorn nous montre qu'il existe un ouvert maximal $W \subset T$ au-dessus duquel $\alpha$ peut être prolongé en courant localement résiduel $\tilde{\alpha}$. $T$ étant connexe, il suffit pour montrer $W=T$ de montrer que $W$ est fermé. Supposons que ce ne soit pas le cas, et $x \in \bar{W} \backslash W$. Soit $V \subset T$ 
une boule ouverte contenant $x$, avec des coordonnées holomorphes $x_{1}, \ldots, x_{n}$. On peut alors écrire $\alpha$ sous la forme : $\alpha:=\sum_{I} \alpha_{I} d x^{I}$, où les multiindices $I$ sont des suites d'entiers compris entre 1 et $n$, de longueur $q$ ou $q-1$, et $\alpha_{I}$ un courant localement résiduel ne contenant pas de $d x_{i}$. Pour montrer que $\alpha$ se prolonge sur $V$, il suffit de montrer que les $\alpha_{I}$ se prolongent. Soit donc un multiindice $I$ fixé. Supposons d'abord $|I|=q-1$. On pose $\alpha_{I}^{\prime}=\alpha_{I} \wedge d x^{I^{c}}$, qui est de bidegré $(n+1,1)$; il suffit de prolonger $\alpha_{I}^{\prime}$. On remarque que $\phi_{*}\left(\alpha_{I}^{\prime} y^{k}\right)=$ $\phi_{*}\left(\alpha y^{k} \wedge d x^{I^{c}}\right)=v_{k, I} d x$, qui par hypothèse se prolonge holomorphiquement sur T. Supposons maintenant $|I|=q$. Alors, on pose $\alpha_{I}^{\prime}=\alpha_{I} \wedge d y \wedge d x^{I^{c}}$. Alors, on vérifie que $\phi_{*}\left(\alpha_{I}^{\prime} y^{k}\right)=\phi_{*}\left(\alpha y^{k} \wedge d y \wedge d x^{I^{c}}\right)=w_{k, I} d x$, qui par hypothèse se prolonge holomorphiquement sur $T$. Il suffit donc pour montrer que $\tilde{\alpha}$ se prolonge sur $V$ de le montrer en supposant $\alpha$ de bidegré $(n+1,1)$. Supposons donc $\alpha$ de bidegré $(n+1,1)$. On pose encore $\phi_{*}\left(\alpha y^{k}\right):=u_{k} d x$ sur $V \cap W$. On sait que les $u_{k}$ se prolongent holomorphiquement sur $T$, on note leur prolongement $\tilde{u}_{k}$. On se place en un point $z$ de $V \cap W$. On a un polynôme associé $P$ comme ci-dessus de degré minimal $d$, unitaire, dont les coefficients $a_{i}$ sont des germes de fonctions méromorphes en $z$, et un autre polynôme associé $P^{\prime}=b_{0} y^{d}+b_{1} y^{d-1}+\cdots+b_{d}$ dont les coefficients sont des germes de fonctions holomorphes en $z$, sans diviseur commun.

On considère le système carré d'équations entre les inconnues $\tilde{a}_{i}(1 \leq i \leq$ d) : $\tilde{a}_{1} \tilde{u}_{k+d-1}+\cdots+\tilde{a}_{d} \tilde{u}_{k}=-\tilde{u}_{k+d}, k=0, \ldots, d-1$. Si l'on considère le système à coefficients dans le corps des germes de fonctions méromorphes en $z$, on voit que le système admet une unique solution $\left(a_{1}, \ldots, a_{d}\right)$. Par conséquent, le déterminant du système sur $V$, qui est méromorphe sur $V$, est non identiquement nul en $z$, donc non plus sur $V$. Le système carré d'équations linéaires, à coefficients dans le corps des fonctions méromorphes sur $V$, admet donc une solution $\left(\tilde{a_{1}}, \ldots, \tilde{a_{d}}\right)$ unique, qui doit donc coïncider avec $\left(a_{1}, \ldots, a_{d}\right)$ en $z$. On associe un polynôme $\tilde{P}^{\prime}=\tilde{b}_{0} y^{d}+\tilde{b_{1}} y^{d-1}+\cdots+\tilde{b_{d}}$ à coefficients holomorphes sur $V$, et un polynôme $\tilde{r}:=y^{d-1} \tilde{u_{0}}+y^{d-2}\left(\tilde{b_{0}} u_{1}+\tilde{b_{1}} \tilde{u_{0}}\right)+\cdots+$ $\left(\tilde{b_{0}} u_{d-1}+\tilde{b_{1}} u_{d-2}+\cdots+\tilde{b_{d-1}} \tilde{u_{0}}\right)$ à coefficients holomorphes dans $V$. On peut supposer que $b_{i}$ en $z$ est induit par $\tilde{b_{i}}$. Considérons le courant sur $\phi^{-1}(V)$ : $\beta_{z}=\operatorname{Res}_{\tilde{P}^{\prime}=0} \tilde{r} / \tilde{P}^{\prime} d x \wedge d y$. alors, on a vu ci-dessus que au voisinage de $F_{z}$, on a : $\beta_{z}=\tilde{\alpha}$. De plus, soit $z^{\prime}$ un autre point de $V \cap W$, et soit $\beta_{z^{\prime}}$ le courant obtenu sur $V$. On a sur $V \phi_{*}\left(y^{k}\left(\beta_{z}-\beta_{z^{\prime}}\right)\right)=0$ pour tout $k \in \mathbb{N}$, donc $\beta_{z}=\beta_{z^{\prime}}$ d'après le lemme 5.2. On peut donc définir un courant localement résiduel sur $\phi^{-1}(W \cup V)$, sans composante verticale, qui prolonge le courant $\tilde{\alpha}$ défini sur $\phi^{-1}(W)$. Cela contredit le fait que $W$ etait un ouvert maximal. Si les prolongements $\tilde{v}_{k}$ sont holomorphes pour $0 \leq k \leq d-1$, on peut faire le raisonnement avec l'existence d'un prolongement maximal $\bar{\partial}$-fermé, car le polynôme $\tilde{r}$ de la construction précédente est holomorphe sur $V \times \mathbb{C}$, et donc le prolongement obtenu est $\bar{\partial}$-fermé en dehors de $T_{\infty}$. Mais comme le courant $\alpha$ est supposé au départ n'avoir pas de pôle sur $T_{\infty}$, le prolongement est en fait $\bar{\partial}$-fermé sur $X$.

On peut donc conclure de ce qui précède que dans le cas général du bidegré $(q, 1)$, le courant $\alpha$ se prolonge sur $X$ en un prolongement localement résiduel 
$\tilde{\alpha}$ de même bidegré sans composante verticale. Ce qui précède montre que si $v_{k}, w_{k}(0 \leq k \leq d-1)$ se prolongent holomorphiquement, le prolongement est $\bar{\partial}$-fermé.

De plus, ce prolongement est unique. En effet, soit $\tilde{\alpha}^{\prime}$ un autre prolongement. Alors, $\tilde{\alpha}-\tilde{\alpha}^{\prime}=0$ sur $U$. Soit $S^{\prime} \subset T \times \mathbb{P}^{1}$ le support de cette différence. On a $\phi_{*}\left(\left[S^{\prime}\right]\right)=$ Cte ; cette constante doit être nulle, donc $S^{\prime}$ est vide.

On déduit de ce qui précède le corollaire suivant :

Corollaire 5.6. Soit $Z$ une hypersurface analytique de $T, T^{\prime}:=T \backslash Z$, et $\alpha$ un courant localement résiduel de bidegré $(q, 1)$ sur $\phi^{-1}\left(T^{\prime}\right)$, sans composante verticale, et $\bar{\partial}$-fermé. Soit d le degré de son polynôme associé. On suppose que $v_{k}:=\phi_{*}\left(\alpha y^{k}\right)$, $w_{k}:=\phi_{*}\left(\alpha y^{k} \wedge d y\right)$ pour tout $k, 0 \leq k \leq d-1$ se prolongent méromorphiquement sur $T$ à travers $Z$. Alors, $\alpha$ se prolonge de manière unique en un courant localement résiduel $\tilde{\alpha}$ de même bidegré sans composante verticale sur $X$. De plus, si le prolongement des $v_{k}, w_{k}(0 \leq k \leq d-1)$ est holomorphe, $\tilde{\alpha} \bar{\partial}$-fermé.

\section{Les théorèmes de Griffiths et d'Henkin-Passare généralisés}

\subsection{La transformation de Radon-Martineau}

Soit $G(p, N)$ la grassmanienne des $p$-plans complexes, et $I \subset G(p, N) \times \mathbb{P}^{N}$ la variété d'incidence, avec les deux projections naturelles $p_{1}: I \rightarrow G(p, N)$ et $p_{2}: I \rightarrow \mathbb{P}^{N}$. On vérifie que $I$ est une fibration en $\mathbb{P}^{p}$ au-dessus de $G(p, N)$ : étant donné $\left(H_{0}, P_{0}\right) \in I$, on se donne un sous-espace linéaire $L=\mathbb{P}^{N-p-1}$ ne rencontrant pas $H_{0}$. On note $G_{L}$ l'ouvert de $G(p, N)$ correspondant aux $H \in G(p, N)$ ne rencontrant pas $L$. Alors, on associe à tout point $(H, P) \in I$ pour tout $H \in G_{P}$, le point $\left(H, H_{0} \cap[L, P]\right)$, où $[L, P]$ est le $\mathbb{P}^{N-p}$ contenant $L$ et $P$. Comme $H_{0}$ ne rencontre pas $L, H_{0} \cap[L, P]$ est au plus un point ; comme $P \in H$ et que $H$ ne rencontre pas $L$, c'est donc un point. On définit ainsi un biholomorphisme entre le voisinage ouvert $p_{1}^{-1}\left(G_{L}\right)$ de $\left(H_{0}, P_{0}\right)$ dans $I$, et $G_{L} \times H_{0}$ (d'inverse $(H, P) \mapsto(H, H \cap[L, P])$, qui transforme $p_{1}$ en une projection canonique sur le premier facteur. En particulier, on voit que $I$ est une variété algébrique lisse.

De même, pour $P \in \mathbb{P}^{N}$, l'ensemble des $\mathbb{P}^{p}$ passant par $P$ définit un sousespace $\sigma_{P} \subset G(p, N)$, isomorphe à $G(p-1, N-1)$, et $p_{2}$ est une fibration en $G(p-1, N-1)$ : si $\left(H_{0}, P_{0}\right) \in I$ est un point quelconque, avec on se donne un hyperplan $H^{\prime}$ ne passant pas par $P_{0}$. Alors, on associe à tout point $(H, P) \in I$ tel que $P \notin H^{\prime}$ le point $\left(H \cap H^{\prime}, P\right) \in G(p-1, N-1) \times \mathbb{P}^{N} \backslash H^{\prime}$. On définit ainsi un morphisme, d'inverse $\left(H^{\prime \prime}, P\right) \mapsto\left(\left[H^{\prime \prime}, P\right], P\right)$, qui transforme $p_{2}$ en une projection canonique sur le second facteur.

On se donne des coordonnées affines de la manière suivante. Soit $H^{\prime}$ un hyperplan de $\mathbb{P}^{N}$, et $L$ un $\mathbb{P}^{N-p-1}$ contenu dans $H^{\prime}$. On se donne en dehors de $H^{\prime}$ des coordonnées affines $(x, y)$ avec $x=\left(x_{1}, \ldots, x_{n}\right), y=\left(y_{1}, \ldots, y_{p}\right), n=$ $N-p$, telles que $L=H \cap\{y=C t e\}$. Alors, on associe à tout $H \in G(p, N)$ 
ne rencontrant pas $L$ le point $(a, b) \in \mathbb{C}^{2 n}$ tel que $H$ s'écrive sous la forme :

$$
x_{i}=\sum_{j=1}^{p} a_{i}^{j} y_{j}+b_{i}(1 \leq i \leq n)
$$

définissant ainsi une carte de $G(p, N)$. Sur l'ouvert de $I$ correspondant aux couples $(H, P)$ avec $H$ ne rencontrant pas $L$ et $P$ en dehors de $H^{\prime}$, on a deux systèmes de coordonnées naturelles :

$(a, x, y)$, pour lequel $p_{2}$ s'écrit $(a, x, y) \mapsto(x, y)$, et

$(a, b, y)$, pour lequel $p_{1}$ s'écrit $(a, b, y) \mapsto(a, b)$; on passe de l'un à l'autre par les relations $x_{i}=\sum_{j=1}^{p} a_{i}^{j} y_{j}+b_{i}$.

Etant donné un ouvert $U$ de $G(p, N)$, l'ouvert $U^{*}:=\cup_{t \in U} H_{t} \subset \mathbb{P}^{N}$ sera dit linéairement $p$-concave (ou linéairement concave pour $p=1$ ). Le complément est dit : linéairement p-convexe. On note $U^{*^{\prime}} \subset G(p, N)$ le domaine correspondant aux $p$-plans contenus dans $U^{*}$. Le complément de $U^{* \prime}$ est réunion de sous-espaces de $G(p, N)$ de la forme $\sigma_{S}$, correspondant aux $H \in G(p, N)$ passant par un point $S$ du complément de $U^{*}$; on voit donc que $U^{* \prime}$ possède un certain type de convexité, qu'on appelle convexité $p$-linéaire (ou convexité linéaire si $p=N-1$ ). Alors, $U^{* \prime}$ est l'enveloppe $p$-linéairement convexe de $U$. On pose $I_{U}:=p_{1}^{-1}(U)$; on note encore $p_{1}$ et $p_{2}$ les deux projections standard $p_{1}: I_{U} \rightarrow U$ et $p_{2}: I_{U} \rightarrow U^{*}$. Par l'axiome du choix, il existe une section $s$ de $p_{2}$, i.e. une application $s: U^{*} \rightarrow I_{U}$ telle que $p_{2} \circ s=I d_{U^{*}}$. Mais il n'existe pas en général de section $s$ continue. On dit que $U^{*}$ est fortement linéairement $p$-concave, si on peut trouver une telle section $s: U \rightarrow I_{U}$ continue. Soit $Z_{0}=\cdots=Z_{N-p-1}=0$ l'équation d'un $p$-plan $H$ donné, avec un système de coordonnées homogènes $\left(Z_{0}, \ldots, Z_{N}\right)$. Les domaines $U_{\epsilon}$ définis par $U_{\epsilon}:=\left\{\left(Z_{0}: \cdots: Z_{N}\right) /\left|Z_{0}\right|^{2}+\cdots+\left|Z_{N-p-1}\right|^{2}-\right.$ $\left.\epsilon\left(\left|Z_{N-p}\right|^{2}+\cdots+\left|Z_{N}\right|^{2}\right)<0\right\}$, forment pour $\epsilon>0$ un système fondamental de voisinages fortement $p$-concaves de $H$.

Les projections $p_{1}$ et $p_{2}$ sont évidemment encore des submersions sur $I_{U}$; de plus, $p_{1}: I_{U} \rightarrow U$ est encore propre, les fibres étant inchangées. On peut donc définir la transformation d'Abel-Radon $\mathcal{R}$ pour la famille des $p$-plans définie par $I_{U}$, qu'on appelle dans ce cas particulier transformation de Radon-Martineau.

\subsection{Une expression explicite de la transformation de Radon-Martineau pour les courants localement résiduels}

On suppose pour simplifier $p=1$. Soit $\alpha$ un courant localement résiduel de bidegré $(N, 1)$ sur $U^{*}$, de support $S$. On se place au voisinage d'une droite $H_{0}$, de sorte qu'on trouve un $L=\mathbb{P}^{N-2}$ ne rencontrant pas $H$. Alors, on a des coordonnées affines naturelles, $a, b \in \mathbb{C}^{2 n}$. De plus, on choisit un hyperplan $H^{\prime}$ à l'infini contenant $L$, évitant $S \cap H_{0}$, d'où des coordonnées affines $\left(x_{1}, \ldots, x_{n}, y\right)$, et des équations pour les droites au voisinage de $H_{0}$ sous la forme $l_{i}(x, y):=a_{i} y+b_{i}-x_{i}=0(1 \leq i \leq n)$. 
Lemme 6.1. Supposons que $H_{0}$ ne rencontre pas le lieu polaire de $\alpha$. Alors, on a au voisinage de $H_{0}$ dans $U: \mathcal{R}(\alpha)=\sum_{I} u_{\operatorname{card}(I)}(a, b) d a^{I} \wedge d b^{I^{c}}$, avec:

$$
u_{k}(a, b):=\sum_{Q \in S \cap H} \operatorname{Res}_{Q}\left[\begin{array}{c}
y^{k} \alpha \\
l_{1}, \ldots, l_{n}
\end{array}\right]:=\sum_{Q \in S \cap H} \operatorname{Res}_{Q}\left[\begin{array}{c}
y^{k} \omega \\
P, l_{1}, \ldots, l_{n}
\end{array}\right]
$$

où l'on note $\alpha$ au point $Q$ sous la forme $\operatorname{Res}_{P=0} \omega / P$, avec $\omega$ une $N$-forme holomorphe.

Démonstration. Ecrivons que $\mathcal{R}(\alpha)$ est l'image directe par la projection standard $U^{*} \times U \rightarrow U^{*}$ du courant localement résiduel sur $U^{*} \times U(1 / 2 \pi i)^{n} \alpha \wedge d l_{1} \wedge$ $\cdots \wedge d l_{n} \wedge \bar{\partial}\left[1 / l_{1}\right] \wedge \cdots \wedge \bar{\partial}\left[1 / l_{n}\right]$, avec $l_{i}(x, y)=a_{i} y+b_{i}-x_{i}$. D'après ce qu'on a vu du calcul de l'image directe d'un courant localement résiduel, on obtient l'expression annoncée.

\subsection{Démonstration du théorème 1.3}

On suppose dans ce qui suit $p=1$. On a donc un domaine $U \subset G(1, N)$ et son domaine linéairement 1-concave associé $U^{*}:=\cup_{t \in U} H_{t}$. Soit $\alpha$ un courant $\bar{\partial}$-fermé de bidegré $(q+1,1), 0<q \leq N-1$ dans $U^{*}$.

Lemme 6.2. $\alpha$ est $\bar{\partial}$-exact ssi $\mathcal{R}(\alpha)=0$.

Démonstration. Supposons d'abord que $\alpha$ est une forme lisse. On se donne un recouvrement ouvert $\left(U_{i}\right)$ de $U^{*}$ par des ouverts fortement linéairement 1-concaves. Alors, la formule de représentation intégrale de Henkin-Gindikin (cf. [8]) montre que sur $U_{i}, \mathcal{R}(\alpha)=0$ implique $\alpha=\bar{\partial} \beta_{i}$, pour une $(q+1,0)$ forme lisse $\beta_{i}$. Sur $U_{i} \cap U_{j}$, on a $\bar{\partial}\left(\beta_{i}-\beta_{j}\right)=0$, ce qui montre que $\beta_{i}-\beta_{j}$ est une $(q+1)$-forme holomorphe. D'après le lemme de prolongement analytique ci-dessus, on a donc $\beta_{i}=\beta_{j}$, donc $\alpha=\bar{\partial} \beta$ pour une forme lisse $\beta$. Soit maintenant $\alpha$ un courant. Alors, on sait d'après le théorème de Dolbeault que $\alpha$ admet dans la même classe de cohomologie une forme lisse : $\alpha=\bar{\partial} \beta+\phi$, avec $\beta$ un courant et $\phi$ une forme lisse de bidegré $(q+1,1)$. Alors $\mathcal{R}(\alpha)=0$ implique $\mathcal{R}(\phi)=0$ donc $\phi=\bar{\partial} \mu$, puis $\alpha=\bar{\partial}(\beta+\mu)$.

Supposons maintenant de plus $\alpha$ courant localement résiduel, et $\mathcal{R}(\alpha)=0$. Alors, $\alpha$ est $\bar{\partial}$-exact d'après le lemme précédent : $\alpha=\bar{\partial} \beta$, avec $\beta$ un courant de bidegré $(q+1,0)$. Montrons qu'on peut choisir pour $\beta$ un courant valeur principale. Tout d'abord, en dehors du support $S$ de $\alpha, \beta$ est représenté par une $(q+1)$-forme holomorphe $\Psi$. De plus, d'après le lemme 2.4, on peut prolonger $\Psi$ en une forme méromorphe sur $U^{*}$, qu'on notera encore $\Psi$. Alors, $\alpha-\bar{\partial}[\Psi]$ est un courant localement résiduel de bidegré $(q+1,1)$ à support dans $S$; il s'écrit de plus $\bar{\partial}(\beta-[\Psi])$, avec $\beta-[\Psi]$ un courant de bidegré $(q+1,0)$ à support dans $S$. D'après le théorème d'unicité de [4], on en déduit que $\alpha-\bar{\partial}[\Psi]=0$.

Alors, d'après le théorème de prolongement analytique ci-dessus, on en déduit que $\Psi$ se prolonge en une forme méromorphe $\tilde{\Psi}$ sur $\mathbb{P}^{N}$. $\tilde{\alpha}:=\bar{\partial}[\tilde{\Psi}]$ est alors un prolongement de $\alpha$. De plus, le prolongement est unique. En 
effet, supposons un autre prolongement $\tilde{\alpha}^{\prime}$; soit $Z$ le support de $\tilde{\alpha}-\tilde{\alpha}^{\prime}$; ce support, s'il était non vide, devrait être une hypersurface algébrique, et donc rencontrerait $U^{*}$, ce qui est impossible.

\subsection{Démonstration du théorème 1.4}

On va démontrer le théorème en quatre étapes ; dans les trois premières étape, on se limitera au cas du prolongement holomorphe. Dans une première étape, on supposera que $\tilde{U}$ est contenu dans un ouvert affine $G_{L} \subset G(1, N)$ correspondant aux droites ne rencontrant pas un $\mathbb{P}^{N-2}$ fixé, et s'écrit avec les coordonées affines $(a, b)$ associées sous la forme $D_{1} \times D_{2}$, avec $D_{1}$ et $D_{2}$ deux polydisques de $\mathbb{C}^{n}$. Dans une deuxième étape, on va pour $\tilde{U}$ quelconque, définir un opérateur de prolongement de "germes de courants au voisinage d'une droite", associé à un chemin de $\tilde{U}$. Dans une troisième étape, on va montrer que ce prolongement ne dépend pas du chemin choisi. Enfin, on conclura dans une dernière étape pour le cas du prolongement méromorphe.

PREMIÈRE ÉTAPE DE LA DÉmonstration. On suppose dans les trois premières étapes que $\mathcal{R}(\alpha)$ se prolonge holomorphiquement sur $\tilde{U}$. On suppose pour la première étape qu'on peut trouver en dehors de $\tilde{U}^{*}$ une sous-espace linéaire $L=\mathbb{P}^{N-2}$. On prend des coordonnées homogènes $X_{1}, \ldots, X_{n}, Y, Z$, de sorte que $\mathbb{P}^{N-2}$ s'écrit $Y=Z=0$. De plus, on suppose que $Y=0$ et $Z=0$ ne coupent pas le support $S$ de $\alpha$ dans $U^{*}$. On utilise les coordonnées affines $x_{1}=X_{1} / Z, \ldots, x_{n}=X_{n} / Z, y=Y / Z$, et pour toute droite $H$ ne rencontrant pas $\mathbb{P}^{N-2}$, on utilise les coordonnées $a_{1}, \ldots, a_{n}, b_{1}, \ldots, b_{n}$, de sorte que les équations de $H$ s'écrivent en coordonnées affines sous la forme : $x_{i}=a_{i} y+$ $b_{i}(1 \leq i \leq n)$. Soit $a:=\left(a_{1}, \ldots, a_{n}\right)$ et $b:=\left(b_{1}, \ldots, b_{n}\right)$.

On suppose de plus que $\tilde{U}$ est de la forme $D_{1} \times D_{2}$, avec $D_{1}$ et $D_{2}$ deux polydisques de $\mathbb{C}^{n}$. On suppose également que $U$ est un produit $D_{1}^{\prime} \times D_{2}^{\prime}$ de polydisques, avec $D_{1}^{\prime} \subset D_{1}$ et $D_{2}^{\prime} \subset D_{2}$.

On définit sur $U$ les fonctions méromorphes $u_{k}(a, b)(k \in \mathbb{Z})$ et $a_{i}(a, b)(1 \leq$ $i \leq d)$ de la manière suivante : tout d'abord, $u_{k}(a, b)$ est le coefficient de $d \bar{b}$ dans $\mathcal{R}\left(\alpha y^{k}\right)$ ( $\alpha y^{k}$ est bien défini pour tout $k \in \mathbb{N}$ en vertu du fait que $Y=0$ et $Z=0$ ne coupent pas le support de $\alpha$ ). On définit $\beta$ la restriction de $p_{2}^{*}(\alpha)$ à $I_{U}=p_{1}^{-1}(U) \simeq U \times \mathbb{P}^{1}$. Alors, on définit le polynôme $P(y)=$ $y^{d}+a_{1}(a, b) y^{d-1}+\cdots+a_{d}(a, b)$, à coefficients méromorphes sur $U$, comme le polynôme unitaire de degré minimal annullant $\beta$ sur $I_{U}$.

Si l'on prend l'image directe par $p_{1}$ de $P(y) y^{k} \beta=0$, on obtient : $u_{k+d}+$ $a_{1} u_{k+d-1}+\cdots+u_{k}=0$, égalité valable pour tout $k \in \mathbb{Z}$. Par ailleurs, on voit comme ci-dessus que le déterminant $\left(u_{l+d-1-j+k}\right)_{0 \leq j, k \leq d-1}$ est non nul pour $l \in \mathbb{Z}$.

On a sur $U^{*}$, d'après le lemme 6.1 :

$$
(\forall k \in \mathbb{Z}) \mathcal{R}\left(\alpha y^{k}\right)=\sum_{I \subset\{1, \ldots, n\}} u_{k+\operatorname{card}(I)} d a^{I} \wedge d b^{I^{c}}
$$


D'autre part, cette $n$-forme méromorphe $\mathcal{R}\left(\alpha y^{k}\right)$ est $d$-fermée sur $U$. Si l'on écrit que le coefficient de $d b_{1} \wedge \cdots \wedge d b_{n} \wedge d a_{i}$ est nul, on obtient sur $U$ :

$$
\frac{\partial}{\partial a_{i}} u_{k}=\frac{\partial}{\partial b_{i}} u_{k+1}(1 \leq i \leq n)
$$

égalité valide pour tout $k \in \mathbb{Z}$.

Lemme 6.3. Pour tout $k \in \mathbb{Z}, u_{k}(a, b)$ se prolongent méromorphiquement sur $\tilde{U}$ en $\tilde{u}_{k}(a, b)$.

Démonstration. Par hypothèse, $\mathcal{R}(\alpha)$ se prolonge holomorphiquement sur $\tilde{U}$; l'expression explicite de $\mathcal{R}(\alpha)$ nous montre donc que déjà que $u_{0}, \ldots, u_{n}$ se prolongent holomorphiquement dans $\tilde{U}$. Montrons tout d'abord par récurrence que tous les $u_{k}, k \leq 0$ se prolongent holomorphiquement sur $D_{1} \times D_{2}^{\prime}$. Supposons que l'on ait prolongé $u_{k}$ holomorphiquement en $\tilde{u}_{k}$ sur $D_{1} \times D_{2}^{\prime}$, pour un certain entier $k \leq 0$. Considérons la 1-forme différentielle holomorphe sur $D_{1} \times D_{2}^{\prime}$ :

$$
\phi:=\sum_{i=1}^{n} \partial_{b_{i}} u_{k} d a_{i} .
$$

Les équations 6.4 nous montrent que sur $D_{1}^{\prime} \times\{b\}$, pour $b \in D_{2}^{\prime}$, on a $\phi=d u_{k-1}$. Comme $\phi$ est holomorphe, on a $d \phi=0$ sur $D_{1} \times\{b\}$ (qui est connexe). Comme de plus $D_{1}$ est simplement connexe, $\phi$ y est donc $d$-exacte : $\phi=d w$, avec $w$ une fonction lisse sur $D_{1} \times\{b\}$, qui en fait est holomorphe (puisque $\phi$ est de type $(1,0))$. Mais $C:=u_{k-1}-w$ est localement constant sur $D_{1}^{\prime} \times\{b\}$, puis constant par connexité. On en déduit que $\tilde{u}_{k-1}(\bullet, b):=w+C$ est un prolongement holomorphe de $u_{k-1}(\bullet, b)$ sur $D_{1} \times\{b\}$. On en déduit, en faisant varier $b \in D_{2}^{\prime}$, une fonction $\tilde{u}_{k-1}(a, b)$ sur $D_{1} \times D_{2}^{\prime}$. D'après un lemme de prolongement analytique ci-dessus $(4.1), \tilde{u}_{k-1}(a, b)$ est en fait holomorphe sur $D_{1} \times D_{2}^{\prime}$. Par récurrence, pour tout $k \leq 0, u_{k}$ se prolongent holomorphiquement en $\tilde{u}_{k}$ sur $D_{1} \times D_{2}^{\prime}$. Or, on sait que pour tout $l \in \mathbb{Z}$, le système carré

$$
b_{1} \tilde{u}_{k+d-1}+\cdots+b_{d} \tilde{u}_{k}=-u_{k+d}(k=l, \ldots, l+d-1)
$$

pour les $b_{i}$ méromorphes sur $U$ a un déterminant non nul sur $U$. Mais si l'on prend $l \leq 2-2 d$, on voit que ce déterminant se prolonge méromorphiquement sur $D_{1} \times \tilde{D}_{2}^{\prime}$, où il ne peut donc pas non plus s'annuler identiquement. Ce système carré admet donc une solution unique $\left(\tilde{a}_{1}, \ldots, \tilde{a}_{d}\right)$ sur $D_{1} \times D_{2}^{\prime}$. Pour tout $i, 1 \leq i \leq n, \tilde{a}_{i}$ doit être un prolongement méromorphe de $a_{i}$. Les équations :

$$
(\forall k \in \mathbb{N}) u_{k+d}+\tilde{a}_{1} u_{k+d-1}+\cdots+\tilde{a}_{d} u_{k}=0
$$

nous montrent alors également l'existence d'un prolongement méromorphe $\tilde{u}_{k}$ des $u_{k}$ sur $D_{1} \times D_{2}^{\prime}$ pour $k \geq 0$.

On va montrer maintenant par récurrence que tous les $\tilde{u}_{k}, k \leq 0$ se prolongent méromorphiquement sur $D_{1} \times D_{2}$. Tout d'abord, les équations $\frac{\partial}{\partial a_{i}} \tilde{u}_{k}=$ 
$\frac{\partial}{\partial b_{i}} \tilde{u}_{k+1}(1 \leq i \leq n)$ restent par méromorphie, vérifiées sur $D_{1} \times D_{2}^{\prime}$. Par ailleurs, les pôles des $\tilde{u}_{k}, k \geq 0$ correspondent à des valeurs où l'un des coefficients $\tilde{a}_{i}$ admet un pôle, c'est-à-dire où la droite $H_{a, b}$ rencontre l'hyperplan à l'infini $H^{\prime}$ en un certain point ; autrement dit, ce lieu polaire est filtré par des $\{a\} \times D_{2}^{\prime}$. Supposons qu'on ait déjà prolongé $\tilde{u}_{k}$ méromorphiquement. Choisissons une valeur de $a \in D_{1}$, en dehors du lieu polaire des $a_{i}$. Alors, on voit, par un raisonnement déjà utilisé, que $\tilde{u}_{k+1}$ se prolonge holomorphiquement sur $\{a\} \times D_{2}$. Par multiplication par un polynôme $Q(a)$ s'annulant à un ordre suffisant aux valeurs de $a$ polaires pour $\tilde{u}_{k+1}$, on voit, toujours d'après le lemme 4.1 , que $\tilde{u}_{k+1}$ se prolonge méromorphiquement sur $D_{1} \times D_{2}$. On a donc un prolongement méromorphe des $\tilde{u}_{k}, k \geq 0$ sur $D_{1} \times D_{2}$, noté encore $\tilde{u}_{k}$. Le système carré

$$
b_{1} \tilde{u}_{k+d-1}+\cdots+b_{d} \tilde{u}_{k}=-\tilde{u}_{k+d}(k=0, \ldots, d-1)
$$

qui admet sur $D_{1}^{\prime} \times D_{2}^{\prime}$ l'unique solution $\left(a_{1}, \ldots, a_{d}\right)$, admet donc sur $D_{1} \times D_{2}$ une unique solution méromorphe $\left(\tilde{a}_{1}, \ldots, \tilde{a}_{d}\right)$. Pour chaque $i, 1 \leq i \leq d, \tilde{a}_{i}$ doit être un prolongement méromorphe de $a_{i}$. Les équations

$$
\tilde{u}_{k+d}+\tilde{a}_{1} \tilde{u}_{k+d-1}+\cdots+\tilde{a}_{d} \tilde{u}_{k}=0(k \in \mathbb{Z})
$$

nous donne alors un prolongement des $u_{k}, k \leq 0$ sur $D_{1}^{\prime} \times D_{2}^{\prime}$.

On déduit de ce qui précède :

Lemme 6.4. Le courant localement résiduel sur l'ouvert de $I I_{U}:=p_{1}^{-1}(U)$ défini $\operatorname{par} \beta:=p_{2}^{*}(\alpha)$, admet un prolongement unique en courant localement résiduel sur $I_{\tilde{U}}:=p_{1}^{-1}(\tilde{U})$.

Démonstration. On applique le théorème des traces énoncé ci-dessus. Identifions $I_{\tilde{U}}$ avec $\tilde{U} \times \mathbb{P}^{1}$, à travers les coordonnées $(a, b,(Y: Z))$. Les conditions du théorème sont vérifiées : toutes les traces se prolongent, les $v_{k}=p_{1 *}\left(\beta y^{k}\right)=$ $\mathcal{R}\left(\alpha y^{k}\right)$, et par ailleurs $\beta \wedge d y=p_{2}^{*}(\alpha \wedge d y)=0$. Par conséquent, $\beta$ se prolonge de manière unique en un courant sur $I_{\tilde{U}} \simeq \tilde{U} \times \mathbb{P}^{1}$.

On peut maintenant prolonger le courant $\alpha$ sur $\tilde{U}^{*}$. Tout d'abord, considérons l'hypersurface de $I_{\tilde{U}} \simeq \tilde{U} \times \mathbb{P}^{1}$ définie par l'équation : $P(a, b, y):=y^{d}+$ $\tilde{a}_{1}(a, b) y^{d-1}+\cdots+\tilde{a}_{d}(a, b)=0$. Cette équation, lorsqu'on qu'on remplace $b$ par $x-a y$, s'écrit $P(a, x-a y, y)=y^{d}+\tilde{a}_{1}(a, x-a y) y^{d-1}+\cdots+\tilde{a}_{d}(a, x-$ $a y)=0$. Si l'on se place au-dessus d'un point $(x, y) \in U$, on voit que cette équation ne dépend pas de $a$. On a donc ainsi défini une hypersurface $\tilde{T}$ de $\tilde{U}$, prolongement du support $T$ de $\alpha$ dans $U$. On a d'après ce qui précède une expression explicite de $\tilde{\beta}$, avec les coordonnées $(a, b, y): \tilde{\beta}=\operatorname{Res}_{I_{\tilde{T}}} \Psi$, avec $\Psi=f_{a, b}(y) / P(a, b, y) d y$, avec $f_{a, b}(y)$ un polynôme de degré $d-1$ dont les coefficients se calculent à l'aide des $\mathcal{R}\left(\alpha y^{k}\right), k \geq 0$. Le fait que $\tilde{\beta}=p_{2}^{*}(\alpha)$ sur $p_{1}^{-1}(U)$ nous montre de même que $\operatorname{Res}_{P(a, a x-y, y)=0} f_{a, b}(y) / P(a, b, y) d y$ nous donne pour $a$ fixé, au voisinage d'un point $(x, y) \in \tilde{U}$, un courant ne dépendant 
pas de $a$. On a ainsi défini, dans le cas du prolongement holomorphe, un courant localement résiduel $\tilde{\alpha}$ sur $\tilde{U}$ prolongeant $\alpha$.

DeuXième ÉtAPe : PROLOngement Le LONG D’un Chemin DE $\tilde{U}^{*}$. Soit $t_{0}$ et $t_{1}$ deux points de $\tilde{U}^{*}$. Par connexité, on peut trouver dans $\tilde{U}^{*}$ un chemin lisse $\gamma$ joignant $t_{0}$ et $t_{1}$. On se donne alors un recouvrement de $\gamma$ par un nombre fini d'ouverts de la forme $U_{i}:=D_{1}^{i} \times D_{2}^{i}(1 \leq i \leq s)$, avec $D_{1}^{i} \cap D_{1}^{j}=\emptyset$ (resp. $\left.D_{2}^{i} \cap D_{2}^{j}=\emptyset\right)$ si $|i-j| \geq 2$. La première étape nous montre alors qu'on peut prolonger $\alpha$ sur l'ouvert de $\mathbb{P}^{N}$ correspondant à la réunion des $U_{i}$. On remarque que l'opérateur qui à un "germe" de courant localement résiduel au voisinage de $H_{t_{0}}$ associe le germe de courant au voisinage de $H_{t_{1}}$ associé à un chemin reliant $t_{0}$ et $t_{1}$ par le procédé décrit dans la deuxième étape est linéaire (i.e. il associe à la somme de deux "germes" la somme des deux images).

TroisiÈme ÉTAPE : MONODROMIE. Supposons donnés deux prolongements $\alpha_{1}$ et $\alpha_{2}$ de $\alpha$ au voisinage de $H_{t_{1}}$, correspondant à deux chemins $\gamma_{1}$ et et $\gamma_{2}$. Pour montrer que $\alpha_{1}=\alpha_{2}$, il suffit par linéarité de montrer que pour le chemin fermé $\gamma$ obtenu en parcourant successivement $\gamma_{1}$, puis $\gamma_{2}$ en sens inverse, l'opérateur linéaire associé au chemin $\gamma$ défini sur les germes de courants résiduels au voisinage de $H_{t_{0}}$, est l'identité. Soit $\gamma^{*}$ cet opérateur. On a $\mathcal{R}\left(\alpha-\gamma^{*}(\alpha)\right)=0$ $\mathrm{au}$ voisinage de $t_{0}$. D'après le théorème de Griffiths généralisé démontré cidessus, on en déduit que $\beta:=\alpha-\gamma^{*}(\alpha)$ est un germe de courant algébrique, i.e. s'écrivant sous la forme $\bar{\partial}[\Psi]$, avec $\Psi$ une $N$-forme rationnelle. Notons les deux faits importants suivants : premièrement, $\gamma^{*}$ conserve le degré $d$ du polynôme $P_{a, b}(y)$ associé. Deuxièmement, $\gamma^{*}$ est visiblement réduit à l'identité pour les germes de courants algébriques. Par conséquent, en itérant $r$ fois l'opérateur $\gamma^{*}$ sur $\alpha$, on obtient le germe de courant $\alpha+r \beta$. Ce germe de courant devant donner comme degré du polynôme $P(a, b, y)$ associé la même valeur, on en déduit que $\beta=0$.

On a donc prolongé le courant $\alpha$ sur tout $\tilde{U}$.

QuATRIÈME ÉTAPE. Examinons ce qui se passe dans le cas d'un prolongement méromorphe. On suppose maintenant juste que $\mathcal{R}(\alpha)$ se prolonge méromorphiquement. Soit $Z \subset \tilde{U}$ le lieu polaire de $\mathcal{R}(\alpha)$. On a d'après ce qui précède prolongé $\alpha$ en un courant localement résiduel $\bar{\partial}$-fermé sur $(\tilde{U}-Z)^{*}$. Il découle de ce qu'on a déjà vu que tous les $a_{i}(1 \leq i \leq d), u_{k}(k \in \mathbb{Z})$ se prolongent holomorphiquement sur $\tilde{U}-Z$. D'autre part, on sait que $u_{0}, \ldots, u_{n}$ se prolongent méromorphiquement à travers $Z$. On va montrer par récurrence que tous les $u_{k}, k \geq 0$ se prolongent à travers $Z$. Supposons qu'on ait montré que $u_{k}$ se prolonge. On a vu alors que pour $a=C t e, d u_{k+1}$ se prolonge. Le lemme suivant nous permet de voir que en fait $u_{k+1}$ se prolonge. Le théorème des traces permet alors de conclure que $\beta$ se prolonge sur $I_{\tilde{U}}$. Le même raisonnement que ci-dessus montre alors que $\alpha$ se prolonge sur $\tilde{U}^{*}$.

Lemme 6.5. Soit $D$ un domaine de $\mathbb{C}^{n}$. Considérons une fonction holomorphe $f$ en dehors d'une hypersurface analytique $T \subset D$. Si $d f$ est méromorphe sur $D, f$ se prolonge méromorphiquement sur D. 
Démonstration. La question est locale. D'après le théorème de Hartogs, il suffit de prolonger méromorphiquement $f$ au voisinage des points réguliers de $T$. On peut donc supposer $T$ de la forme $\{y=0\}$, avec des coordonées affines $\left(x_{1}, \ldots, x_{n-1}, y\right)$. On peut supposer $f$ holomorphe dans une couronne $K:=D \times\{|z|<\epsilon\} \backslash\{|z| \leq \eta, \eta<\epsilon\}$. Alors, $f$ peut s'écrire sur $K$ comme $f=f_{+}-f_{-}$, avec $f_{+}=\sum_{k \geq 1} u_{-k}(x) y^{k-1}, f_{-}=\sum_{k \geq 0} u_{k}(x) / y^{k+1}$, avec

$$
u_{k}(x):=1 / 2 i \pi \int_{|y|=\rho} f(x, y) y^{k}(\eta<\rho<\epsilon)
$$

holomorphes sur $D$. Dire que $f$ se prolonge méromorphiquement sur $D \times\{|z| \leq$ $\epsilon\}$ revient à dire que $y^{m} f$ se prolonge holomorphiquement pour un certain $m$, donc que $f_{-}$comporte un nombre fini de termes non identiquement nuls. Remarquons grâce à la convergence uniforme, on peut dériver sous le signe somme. On obtient donc $f_{y}(x, y)=\sum_{k \in \mathbb{Z}} u_{-k-1}(x) k y^{k-1}$ sur $K$.

Supposons que $d f$ se prolonge méromorphiquement sur $D \times\{|z| \leq \epsilon\}$. Alors, il en est de même de $f_{z}$. On en déduit que seuls un nombre fini des $u_{i}, i \geq 0$ sont non identiquement nuls. Par conséquent, $f$ aussi se prolonge méromorphiquement.

Remarque. On ne suppose pas l'ouvert $\tilde{U}$ "projectivement convexe" comme dans le théorème d'Henkin-Passare. Mais il découle de notre théorème que si $\mathcal{R}(\alpha)$ se prolonge méromorphiquement dans un domaine $\tilde{U}$, il se prolonge automatiquement dans son "enveloppe linéairement convexe" correspondant aux droites contenues dans $\tilde{U}^{*}$.

Corollaire 6.6. Supposons $\mathcal{R}(\alpha)$ rationnel. Alors, $\alpha$ se prolonge en un courant localement résiduel sur $\mathbb{P}^{N}$.

Corollaire 6.7. Soit $U$ un ouvert linéairement 1-concave de l'espace projectif $\mathbb{P}^{3}$. Supposons que $U$ contient un 2-plan complexe, et $U^{\prime}$ connexe. On se donne un courant $\alpha$ localement résiduel de type $(3,1)$ dans $U$. Alors, $\alpha$ se prolonge dans $\mathbb{P}^{3}$ en courant localement résiduel.

On va pour la démonstration utiliser la pseudoconcavité au sens d'Andreotti.

Lemme 6.8. Soit $V \subset \mathbb{P}^{3}$ un domaine linéairement 1-concave. Supposons que $V$ contienne un plan complexe P. Alors si l'ouvert $V^{\prime} \subset G(1, N)$ est connexe, il est pseudoconcave au sens d'Andreotti.

Démonstration. Soit $Z_{0}=0$ l'équation du 2-plan $P$ contenu dans $V$, pour un système de coordonnées homogènes $\left(Z_{0}, \ldots, Z_{3}\right)$. On a un système fondamental $\left(V_{\epsilon}\right)_{\epsilon>0}$ de voisinages de $P$, disjoints du point $L=\left\{Z_{1}=Z_{2}=Z_{3}=0\right\}$, où $V_{\epsilon}$ est défini par l'inégalité : $\left|Z_{0}\right|^{2}<\epsilon\left(\left|Z_{1}\right|^{2}+\left|Z_{2}\right|^{2}+\left|Z_{3}\right|^{2}\right)$. Soit $V_{\eta}$ un tel domaine contenu dans $V$.

Considérons le domaine associé $V_{\eta}^{\prime} \subset G(1,3)$. On se donne un point de la frontière $t \in \partial V_{\eta}^{\prime}$. Alors, la droite correspondante $H_{t}$ n'est pas contenue dans $V_{\eta}$. Mais une droite arbitrairement proche est contenue dans $V_{\eta} ; H_{t}$ 
est donc contenue dans $\overline{V_{\eta}}$. Par conséquent, $H_{t}$ rencontre $V_{\eta}$ en un certain point $x$. Alors, on peut trouver un plan complexe $P^{\prime}$, contenant la droite $H_{t}$, contenu dans $\overline{V_{\eta}}$, et qui ne coupe le complémentaire de $V_{\eta}$ que en $x$. On se donne un point $y$ de $P^{\prime}$ en dehors de $H_{t}$. Alors, on associe à chaque point $z \in H_{t}$ la droite $D_{z}$ reliant $z$ et $y$. Si $z$ est différent de $x, D_{z}$ est contenue dans $V_{\eta}$, puisque contenue dans $P^{\prime}$ et que $P^{\prime}$ ne rencontre le complémentaire de $V_{\eta}$ que en $x$. On construit ici un $\mathbb{P}^{1}$ dans $G(1,3)$, correspondant aux droites passant par $z$ et contenues dans $P^{\prime}$, contenu dans $\overline{V_{\eta}^{\prime}}$, et qui ne rencontre le complémentaire de $V_{\eta}^{\prime}$ qu'au point $t$. Cela démontre la pseudoconcavité, d'après ce qu'on a vu.

On peut maintenant terminer la démonstration du corollaire. Soit $V$ un domaine linéairement 1 -concave de $\mathbb{P}^{3}$, contenant un plan complexe, avec $V^{\prime}$ connexe. La transformée $\mathcal{R}(\alpha)$ est méromorphe sur $V^{\prime}$. D'après le lemme d'extension 4.5 , comme $V^{\prime}$ est pseudoconcave au sens d'Andreotti, on en déduit que $\mathcal{R}(\alpha)$ est rationnelle. D'après le corollaire 6.6 ci-dessus, $\alpha$ se prolonge sur $\mathbb{P}^{N}$ en un courant localement résiduel.

\section{References}

[1] J.-E BJÖRK, Residues and D-modules, In : "The legacy of Niels Henrik Abel”, The Abel Bicentennial, Oslo, Springer, 2002, 605-651.

[2] N. ColefF and M. HererRa, "Les courants résiduels associés à une forme méromorphe", Springer Lect. Notes 633, 1978.

[3] A. Dickenstein, M. Hererra and C. Sessa, On the global lifting of meromorphic forms, Manuscripta Math. 47 (1984), 31-45.

[4] A. Dickenstein and C. SESSA, Canonical representative in moderate cohomology, Invent. Math. 80 (1985), 417-434.

[5] P. Dingoyan, Un phénomène de Hartogs dans les variétés projectives, Math. Z. 232 (1999), 217-240.

[6] B. FABRE, On a problem of Griffiths : inversion of Abel's theorem for families of zero-cycles, Ark. Mat. 41 (2003), 61-84.

[7] P. GRIFFITHS, Variations on a theorem of Abel, Invent. math. 35 (1976), 321-390.

[8] S. G. Gindikin and G. M. Henkin, Integral geometry for $\bar{\partial}$-cohomology in q-linear concave domains in $C P^{n}$, Functional Anal. Appl. 12 (1978), 247-261.

[9] G. Henkin, The Abel-Radon transform and several complex variables, Ann. of Math. Studies 137 (1995), 223-275.

[10] G. HENKIN and M. PASSARE, Abelian differentials on singular varities and variations on a theorem of Lie-Griffiths, Invent. Math. 135 (1999), 297-328.

[11] G. Henkin, Abel-Radon transform and applications, In : "The legacy of Niels Henrik Abel", Springer, The Abel Bicentennial, Oslo, 2002, 477-494.

[12] M. HERERRA and D. LiEBERMAN, Residues and principal values on complex spaces, Math. Ann. 194 (1971), 259-294. 
[13] H. KNESER, Einfacher Beweis eines Satzes über rationale Funktionen zweier Veränderlichen, Hamburg Univ. Math. Sem. Abhandl. 9 (1933), 195-196.

[14] M. PASSARE, Residues, currents, and their relations to ideals of meromorphic functions, Math. Scand. 62 (1988), 75-152.

[15] R. Remmert and K. Stein, Über die wesentlichen Singularitäten analytischer Mengen, Math. Ann. 126 (1953), 263-306.

[16] W. RothsteIn, Ein neuer Beweis des Hartogsschen Hauptsatzes und seine Ausdehnung auf meromorphe Funktionen, Math. Z. 53 (1950), 84-95.

[17] L. Schwartz, "Théorie des distributions", 2nd ed., Hermann, Paris, 1966.

[18] J. A. Wood, A simple criterion for local hypersurfaces to be algebraic, Duke Math. J. 51 (1984), 235-237.

22, rue Emile Dubois 75014 Paris, France

bfabre@math.su.se 